\title{
Synthesis of Spiro Pyrazolone-Oxindole and Bicyclic Pyrazolone De- rivatives via Solvent-Dependent Regioselective Aza-1,4/1,6-Michael and Intramolecular Cycloaddition under Catalyst-Free Conditions
}

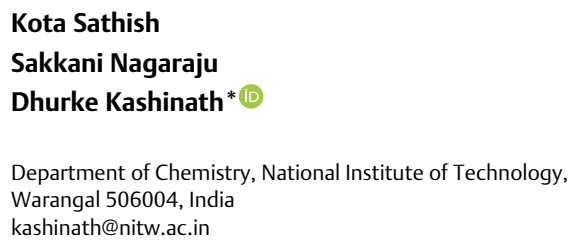

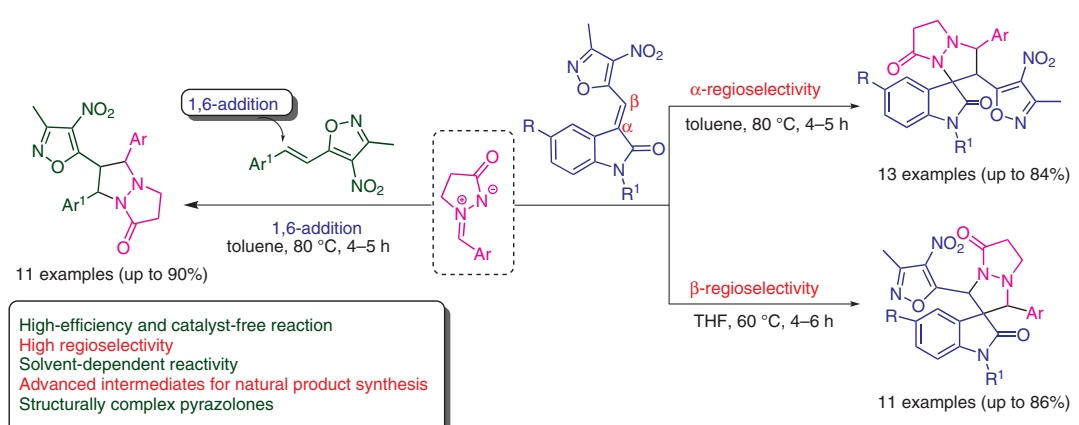

Received: 22.03.2021

Accepted after revision: 12.04 .2021

Published online: 13.04 .2021

DOI: 10.1055/a-1480-9837; Art ID: so-2021-d0016-op

License terms: (C) $९ \$$

(c) 2021. The Author(s). This is an open access article published by Thieme under the terms of the Creative Commons Attribution-NonDerivative-NonCommercial-License, permitting copying and reproduction so long as the original work is given appropriate credit. Contents may not be used for commercial purposes or adapted, remixed, transformed or built upon. (https://creativecommons.org/licenses/by-nc-nd/4.0/)

Abstract A solvent-dependent, highly regioselective [3+2]-cycloaddition reaction of isoxazole-styrenes and azomethine imines under catalyst-free conditions is reported, furnishing a library of pyrazolonespirooxindole hybrids. Good regioselectivity for the isomeric structures was achieved by the reaction of isoxazole-styrene and azomethine imine in different solvents and temperatures. The developed method was extended for the synthesis of tri-substituted dinitrogen-fused pyrazolones by using a 1,6-Michael addition reaction. Furthermore, the isoxazole moiety was converted into a carboxylic acid as a model study via ring opening.

Key words spirooxindole-pyrazolone hybrids, bicyclic pyrazolones, isoxazole-styrenes, switchable 1,3-dipolar cycloaddition, solventdependent reactivity, catalyst-free conditions

Spirooxindoles are structurally complex molecules with a quaternary carbon at $\mathrm{C}-3$ of the indole nucleus, joined by multiply substituted five-membered pyrrolidine rings with defined stereocenters. The spirooxindole skeleton is present in many natural products such as spirotryptostatins, rhynchophyllines, horsfiline, and (+)-elacomines, and members of this class show a wide range of biological properties. Due to their structural complexity and biological prominence, these compounds have attracted the attention of synthetic chemists. As a result, many methods have been developed over the years for their synthesis. The [3+2]-cycloaddition and tandem (one-pot), multicomponent reactions of isatin or its derivatives in the presence of metal-based and organocatalysis are some of the common methods employed. $^{1 \mathrm{a}-\mathrm{c}}$ In many cases, the synthesized compounds were tested for biological activity and, as a result, this moiety has become a promising scaffold in drug discovery. ${ }^{\text {id }}$

Pyrazolones (pyrazole-5-ones) represent another useful scaffold commonly found in many biologically active molecules. Derivatives of pyrazolones such as morazone, phenazone, phenylbutazone (NSAIDs), tartrazine (anticancer) phenidone, and BW357U (anorectic) are sold as commercial drugs. ${ }^{2}$ Acyl substituted pyrazolones can undergo isomerization (proton exchange) and keto-enol tautomerism. These features make them useful synthons in organic chemistry for electrophilic and nucleophilic addition reactions. ${ }^{3}$ Reports on $\mathrm{N}, \mathrm{N}^{\prime}$-fused bicyclic pyrazolones are rare. They have been reported as $\gamma$-lactam antibiotics, antibacterial agents, ${ }^{4 a, b}$ acetyl-CoA carboxylase (ACC) inhibitors, ${ }^{4 c}$ sarcoplasmic reticulum $\mathrm{Ca}^{+2}$-ATPase inhibitors, ${ }^{4 \mathrm{~d}}$ and anticancer agents, ${ }^{4 e}$ and they have also been used as herbicides and pesticides (Figure 1$)^{5}$

The development of a regioselective method to access these derivatives is highly desirable, particularly one that allows the generation of complex molecules with structural diversity. ${ }^{6 a}$ In this context, domino-cascade, cycloaddition and chelation controlled reactions have proven to be efficient in the presence of Lewis acids, ${ }^{6 b}$ organocatalysts, ${ }^{6 c, d}$ metal catalysts, ${ }^{6 e}$ and in various solvents. ${ }^{6 f, g}$

Nitrones, $N$-imides and pyridinium ylides are useful intermediates for the synthesis of functionalized pyrrolidines, dihydrooxazoles, and isoxazoles via 1,3-dipolar cycloaddition reactions (Michael addition, followed by Mannich type cyclization). ${ }^{7}$ In particular, azomethine imines (acyclic and 


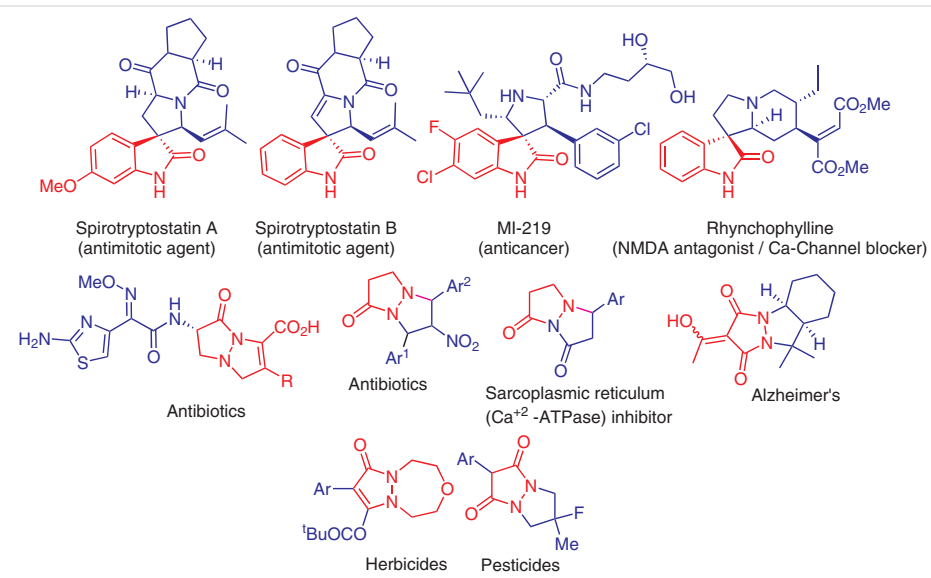

Figure 1 Representative biologically important spirooxindoles and pyrazolidinones

$\mathrm{N}, \mathrm{N}^{\prime}$-cyclic) have been used as 1,3-dipoles for [3+2]cycloaddition reactions $s^{4 a, 8}$ to give complex spirooxindole derivatives. In this connection, spiro[pyrazolidin-3,3'-oxindoles] have been obtained by [3+2]-cycloaddition reaction ( $\beta$-regioselective 1,4-aza Michael addition and intramolecular cyclization) of azomethine imines and methyleneindolinones in the presence of chiral bis-phosphoric acid, ${ }^{9 a} N, N^{\prime}$-dioxide- $\mathrm{Mg}(\mathrm{OTf})_{2},{ }^{\text {gb }}$ or $\mathrm{Cu}(\mathrm{OAc})_{2}{ }^{9 \mathrm{c}}$ as catalysts with high enantioselectivity. In a similar approach, Yan and co-workers reported a catalyst-free method for generating spiro[indoline-3,2'-pyrazolo[1,2-a]pyrazoles] using 3phenacyliceneoxindoles. ${ }^{9 \mathrm{~d}}$

3-Methyl-4-nitro-5-isatylidenyl-isoxazole $(\mathbf{1})^{10 a}$ represents an excellent precursor for our studies, having two reactive cites at the $\alpha$ - and $\beta$-positions to the unsaturated double bond, as shown in Scheme 1. This feature has been applied for use as a dipolarophile for the construction of functionalized 3,3-disubstituted oxindoles and spirocyclic oxindoles (via tandem Michael addition and aldol/Mannich reactions) ${ }^{10 \mathrm{~b}-\mathrm{f}}$ to give the desired products in good yields with excellent regio- and/or stereoselectivity. In a similar study, Liu and co-workers synthesized isoxazole-dispirobisoxindole and bispirocyclic hexahydroxanthones via $\beta$-regioselective [3+2]-cycloaddition and domino Michael-Michael addition reactions using quinine and chiral thioureas as organocatalysts. ${ }^{11}$ In a recent report, Chowhan and coworkers demonstrated an unusual $\mathrm{C}-\mathrm{N}-\mathrm{C}[3+2]$-cycloaddition of 3-methyl-4-nitro-5-styrylisoxazole and isatin N,N'cyclic azomethine imines with high diastereoselectivity. ${ }^{12}$ All these methods have their own advantages in terms of regioselectivity, stereoselectivity and product yields. However, a switchable regioselective reaction has never been realized on 3-methyl-4-nitro-5-isatylidenyl-isoxazole (1). Considering the importance of spiro- and bicyclic pyrazolones, we herein report the first example of a solvent-dependent, regioselective-switchable reaction between $\mathrm{N}, \mathrm{N}^{\prime}-$

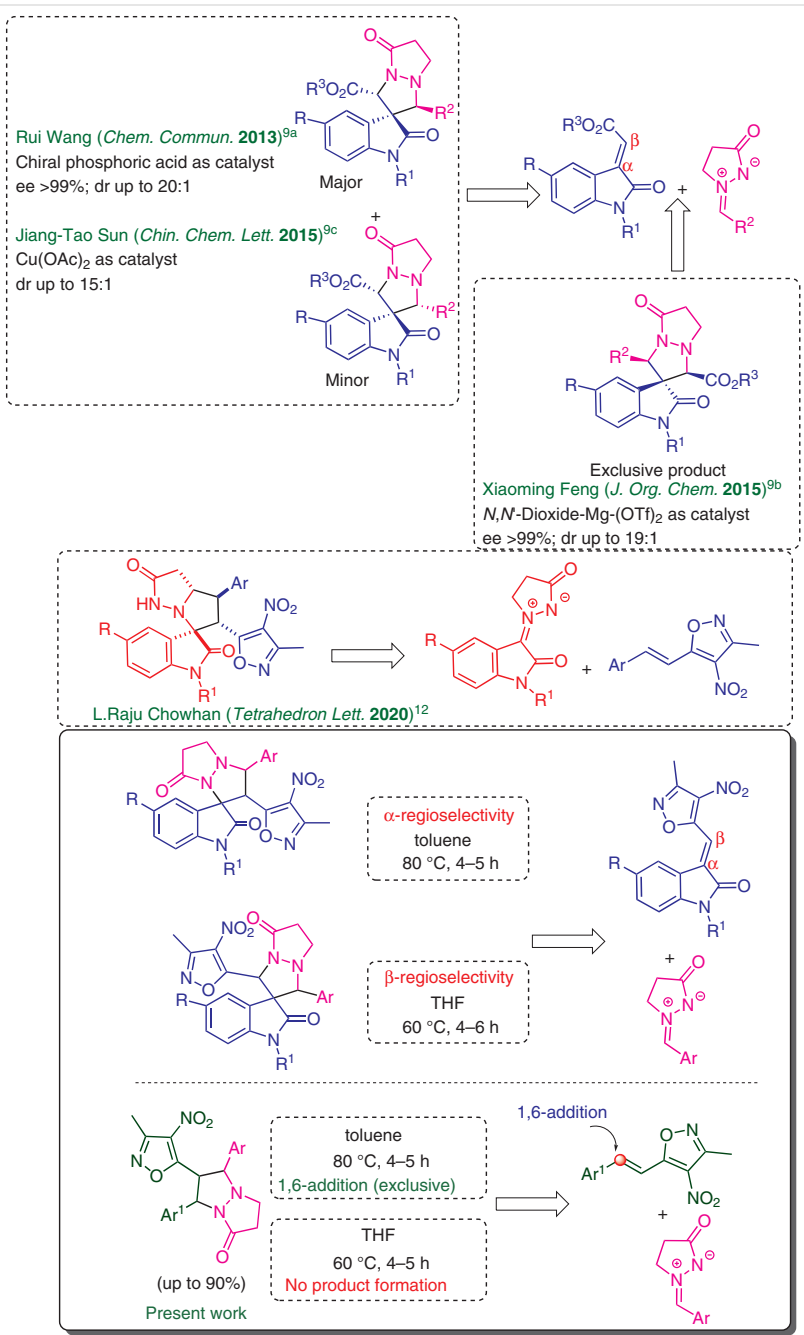

Scheme 1 Reactivity profile of isoxazole-styrenes: reported methods and current strategy 
cyclic azomethine imines and 3-methyl-4-nitro-5-isatylidenyl-isoxazoles leading to complex dinitrogen-fused bicyclic and spirocyclic oxindoles in good yields.

Towards the synthesis of functionalized spirooxindole derivatives, we utilized 3-methyl-4-nitro-5-isatylidenylisoxazole (1a) and azomethine imine (2) as model substrates in dichloromethane (DCM), both in the presence of organic bases (TEA and DABCO) and Lewis acids $\left(\mathrm{Zn}(\mathrm{OTf})_{3}\right.$, $\mathrm{AlCl}_{3}$ ) at room temperature and heating $\left(25-60{ }^{\circ} \mathrm{C}\right)$, but these conditions did not yield the expected products (Table 1 ; entries 1-5). The same reaction was then performed in 1,2-dichloroethane (DCE) at $80{ }^{\circ} \mathrm{C}$ for $12 \mathrm{~h}$ under catalystfree conditions. To our satisfaction, the formation of products 3a and 4a was observed in 25 and 30\% yield, respectively (Scheme 2 and Table 1 , entry 6 ).
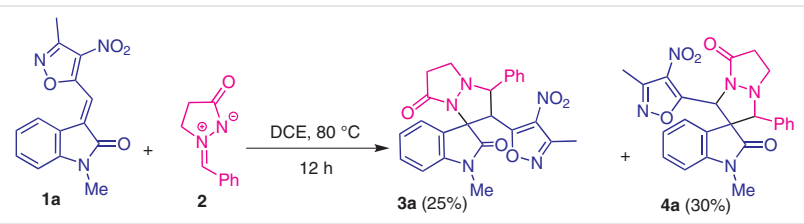

Scheme 2 Synthesis of dinitrogen-fused spirooxindoles

Encouraged by this result, the reaction was carried out in different solvents under catalyst-free conditions; the results are summarized in Table 1 (entries 7-26). It is important to note that the use of polar solvents (DMSO, DMF, $\mathrm{MeCN}, \mathrm{MeOH}, \mathrm{EtOH}$ and water) did not give the products even at elevated temperature. Even for the chlorinated solvents, the overall yield of products $\mathbf{3 a}$ and $\mathbf{4 a}$ could not be related to their boiling points and relative polarity $\left[\mathrm{CH}_{2} \mathrm{Cl}_{2}\right.$ (no product), $\mathrm{DCE}(55 \%)$ and $\mathrm{CHCl}_{3}$ (60\%)]. At this point, the use of other common solvents such as toluene, xylene, ethyl acetate, diethyl ether and tetrahydrofuran (from non-polar to moderately polar) were examined at temperatures between 40 and $80^{\circ} \mathrm{C}$ (without the catalyst). Surprisingly, the reaction in toluene and xylene at $80{ }^{\circ} \mathrm{C}$ gave $3 a$ in 84 and $70 \%$ yield, respectively, as major products, along with 4a in $5-10 \%$ yield as the minor regioisomer (Table 1, entries 14 and 15). On the other hand, reaction in tetrahydrofuran at $60{ }^{\circ} \mathrm{C}$, gave a contrasting outcome, with $4 \mathbf{a}$ formed as the major product (75\%) and 3a as the minor product (15\%) for $4 \mathrm{~h}$ (Table 1, entry 21). Both the isomers were characterized by ${ }^{1} \mathrm{H}$ and ${ }^{13} \mathrm{C}$ NMR spectroscopic and mass spectrometric analyses. The ${ }^{1} \mathrm{H}$ NMR spectrum of compound 3a in $\mathrm{CDCl}_{3}$ included two doublets at $\delta=5.05(\mathrm{~d}, J=10.8 \mathrm{~Hz})$ and 4.49 $(\mathrm{d}, J=10.8 \mathrm{~Hz}) \mathrm{ppm}$, indicating that the phenyl and isoxazole ring protons are on adjacent carbons (Figure 1 in the Supporting Information); whereas for 4a the two characteristic protons appeared as singlets at $\delta=5.97$ and $4.12 \mathrm{ppm}$ due to the phenyl and isoxazole rings being separated. The observed HRMS mass ion of $\mathbf{3 a}(\mathrm{m} / z$ 460.1614 [M+1]) and of 4a $(m / z$ 460.1591 [M+1]) further confirmed the formation of the desired products.
At this juncture, we attempted to understand the solvent effect on the outcome of the reaction. Towards this, different combinations of toluene and tetrahydrofuran were studied (Table 1, entries 22-26). From these observations, we suggest that the polar chelating nature of the tetrahydrofuran helps to increase the electron density on the $\alpha$-carbon of isoxazole-oxindole styrene, which facilitates attack of negatively charged nitrogen of the azomethine imine $\mathbf{2}$ to deliver product $\mathbf{4 a}$ as the major isomer.

Table 1 Optimization of Reaction Conditions ${ }^{a}$

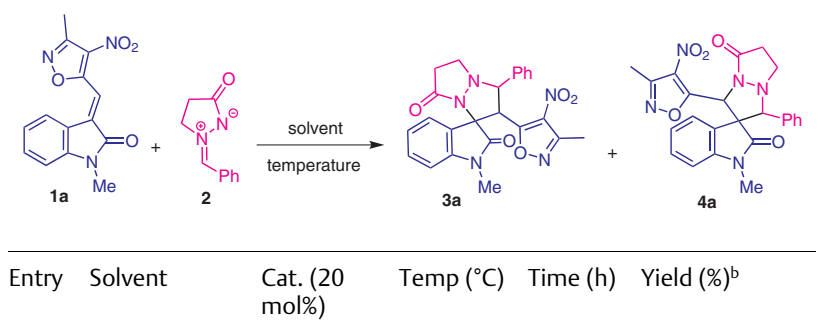

\begin{tabular}{|c|c|c|c|c|c|c|}
\hline & & & & & $3 a$ & $4 a$ \\
\hline 1 & $\mathrm{CH}_{2} \mathrm{Cl}_{2}$ & TEA & $\mathrm{rt}$ & 12 & ND & ND \\
\hline 2 & $\mathrm{CH}_{2} \mathrm{Cl}_{2}$ & DABCO & $\mathrm{rt}$ & 12 & ND & ND \\
\hline 3 & $\mathrm{CH}_{2} \mathrm{Cl}_{2}$ & $\mathrm{Zn}(\mathrm{OTf})_{3}$ & $\mathrm{rt}$ & 12 & ND & ND \\
\hline 4 & $\mathrm{CH}_{2} \mathrm{Cl}_{2}$ & $\mathrm{AlCl}_{3}$ & $\mathrm{rt}$ & 12 & ND & ND \\
\hline 5 & $\mathrm{CH}_{2} \mathrm{Cl}_{2}$ & - & 60 & 12 & ND & ND \\
\hline 6 & DCE & - & 80 & 12 & 25 & 30 \\
\hline 7 & $\mathrm{CHCl}_{3}$ & - & 60 & 12 & 15 & 45 \\
\hline 8 & DMSO & - & 80 & 12 & ND & ND \\
\hline 9 & DMF & - & 80 & 12 & ND & ND \\
\hline 10 & $\mathrm{CH}_{3} \mathrm{CN}$ & - & 80 & 12 & ND & trace \\
\hline 11 & $\mathrm{MeOH}$ & - & 80 & 12 & ND & ND \\
\hline 12 & EtOH & - & 80 & 12 & ND & ND \\
\hline 13 & $\mathrm{H}_{2} \mathrm{O}$ & - & 80 & 12 & ND & ND \\
\hline 14 & toluene & - & 80 & 4 & 84 & 10 \\
\hline 15 & xylene & - & 80 & 4 & 70 & 5 \\
\hline 16 & toluene & - & $\mathrm{rt}$ & 24 & trace & ND \\
\hline 17 & EtOAc & - & 80 & 12 & ND & ND \\
\hline 18 & $\mathrm{Et}_{2} \mathrm{O}$ & - & 40 & 4 & ND & ND \\
\hline 19 & THF & - & 80 & 12 & 20 & 60 \\
\hline 20 & THF & - & $\mathrm{rt}$ & 48 & ND & 60 \\
\hline 21 & THF & - & 60 & 4 & 15 & 75 \\
\hline 22 & THF/Tol (1:1) & - & 80 & 4 & 45 & 30 \\
\hline 23 & THF/Tol (1:2) & - & 80 & 4 & 55 & 20 \\
\hline 24 & THF/Tol (1:3) & - & 80 & 4 & 60 & 15 \\
\hline 25 & THF/Tol (2:1) & - & 80 & 4 & 30 & 40 \\
\hline 26 & THF/Tol (3:1) & - & 80 & 4 & 25 & 55 \\
\hline
\end{tabular}

${ }^{a}$ All reactions were performed with $1 \mathrm{a}(0.35 \mathrm{mmol})$ and $\mathbf{2}(0.35 \mathrm{mmol})$ in solvent $(4 \mathrm{~mL})$.

b Isolated yields. 
After identifying the two optimal reaction conditions, we turned our attention towards the substrate scope of the reactions. Accordingly, variously substituted isatins (substitution on aromatic ring and nitrogen) and azomethine imines (aromatic and heteroaromatic) were reacted under the optimized conditions (both in toluene and THF) to afford the corresponding cycloaddition products $3 \mathbf{a}-\mathbf{m}$ (Scheme 3) and $\mathbf{4 a - k}$ (Scheme 4) in good yields $70-86 \%$ in 4-6 h. All newly synthesized compounds were characterized using ${ }^{1} \mathrm{H},{ }^{13} \mathrm{C}$ NMR spectroscopy and mass spectrometry. Furthermore, single-crystal X-ray crystallographic data were obtained for compounds $\mathbf{3 b}$, 3d, $\mathbf{3 i}$ and $\mathbf{4 c}$, clearly indicating that the isoxazole and phenyl groups are adjacent to each other in products in Scheme 3; whereas in the products in Scheme 4, the rings were assigned opposite each other.
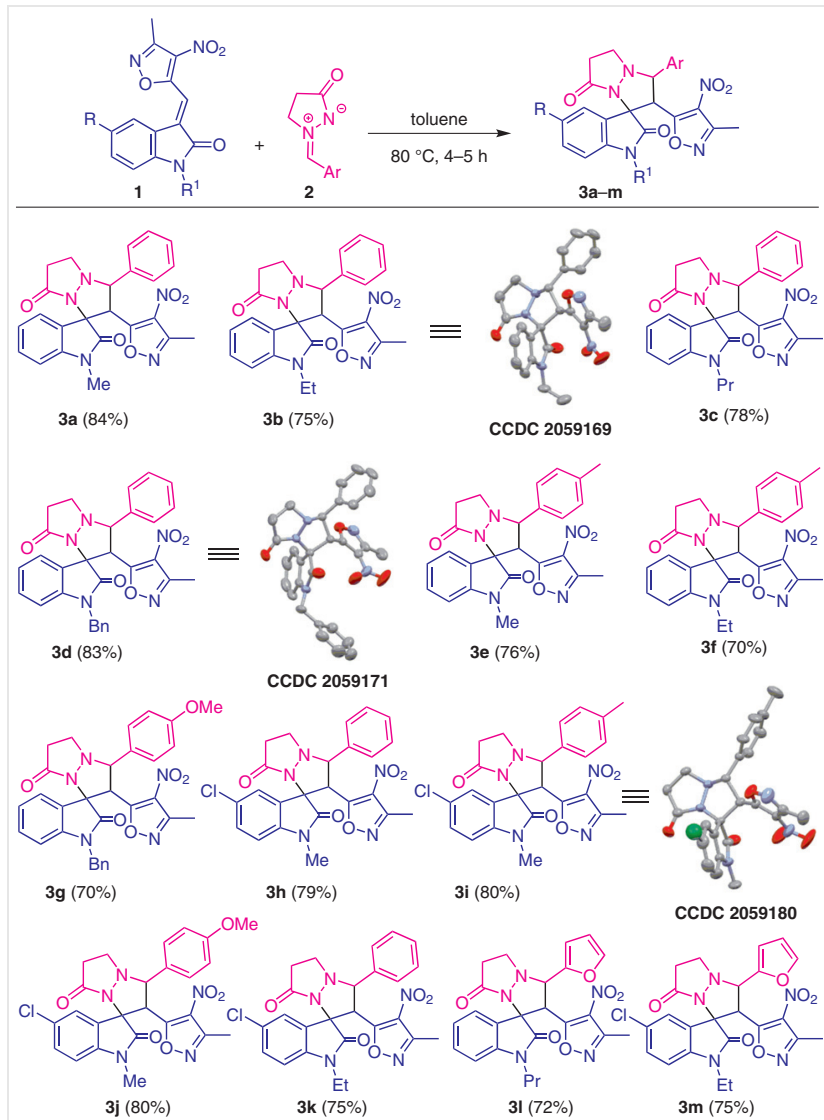

Scheme 3 Substrate scope for the synthesis of functionalized dinitrogen-fused spirooxindoles $3 a-m$

Based on above results and on single-crystal data, a plausible mechanism can be proposed for the [3+2]-cycloaddition reaction (Scheme 5). Azomethine imine 2 reacts with 3-methyl-4-nitro-5-isatylidenyl-isoxazole 1 via aza1,6 Michael addition ( $\alpha$-regioselectivity) to give adduct $\mathbf{I}$. This adduct can undergo intramolecular cyclization to afford desired product 3; in contrast, in THF the same reac-

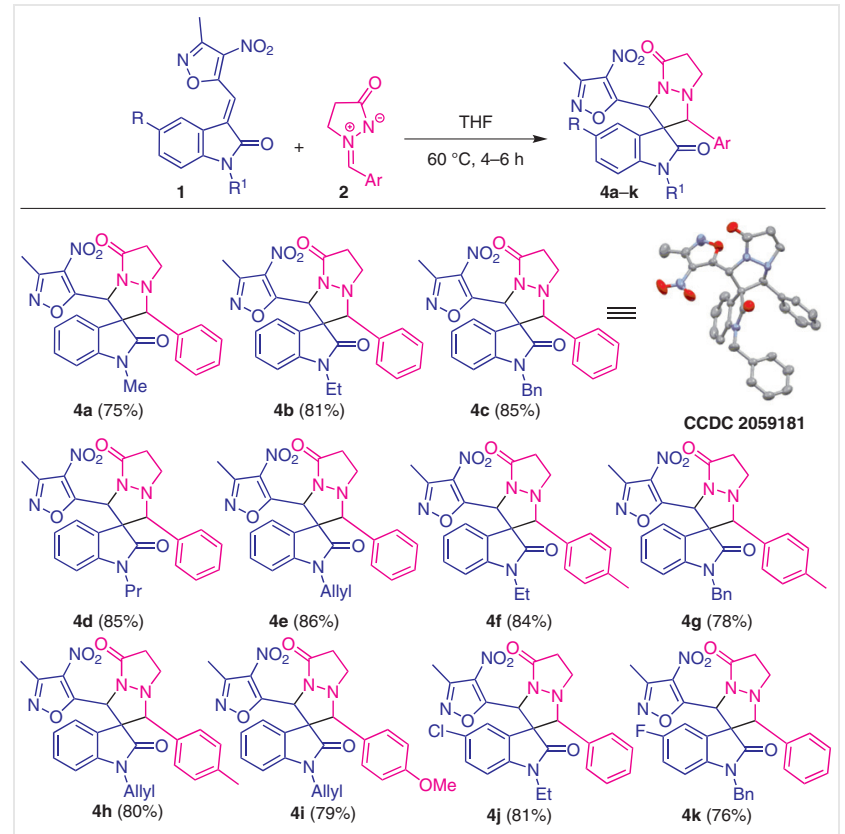

Scheme 4 Substrate scope for the synthesis of functionalized dinitrogen-fused spirooxindoles $\mathbf{4 a - k}$

tion proceeds through aza-1,4 Michael addition ( $\beta$-regioselectivity) followed by intramolecular cyclization to deliver product 4.
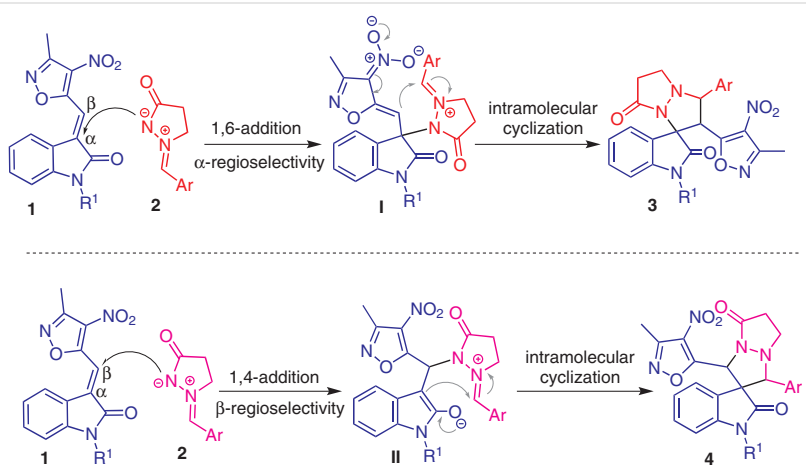

Scheme 5 Proposed mechanism for the two cycloaddition pathways

Considering the biological importance of spirooxindoles, isoxazoles and pyrazolones (Figure 1), we extended our strategy to the synthesis of diphenyltetrahydropyrazolo pyrazolones (dinitrogen-fused heterocycles). To achieve this, isoxazole-styrenes $\mathbf{5}$ were treated with azomethine imines 2 under the optimized reaction conditions (in toluene and THF). To our surprise, the reaction was successful only in toluene, delivering isoxazole-based dinitrogenfused compounds $\mathbf{6 a}-\mathbf{k}$ in good yields of $65-90 \%$ in $4-5$ hours (Scheme 6). Similar to the above mechanism, in this case the desired compounds $\mathbf{6}$ were also formed by the reaction of isoxazole styrene $\mathbf{5}$ with azomethine imine $\mathbf{2}$ to afford adduct III via aza-1,6-Michael addition followed by 
intramolecular cyclization. The isoxazole moiety was also used as a masked ester to generate carboxylic acid via ring opening under basic-oxidative conditions. ${ }^{13}$ Finally the cycloadducts $\mathbf{3 a}$ and $\mathbf{6 b}$ were converted into carboxylic acids $7 \mathbf{a}$ and $\mathbf{8 b}$ in 78 and $85 \%$ yield, respectively, by treatment with aq. $\mathrm{NaOH}$ (Scheme 7 and Scheme 8). The carboxylic acid $\mathbf{8 b}$ was then further functionalized into ester $\mathbf{9 b}$ and amide 10b derivatives in 65 and $85 \%$ yield, respectively, under the standard conditions (Scheme 8).
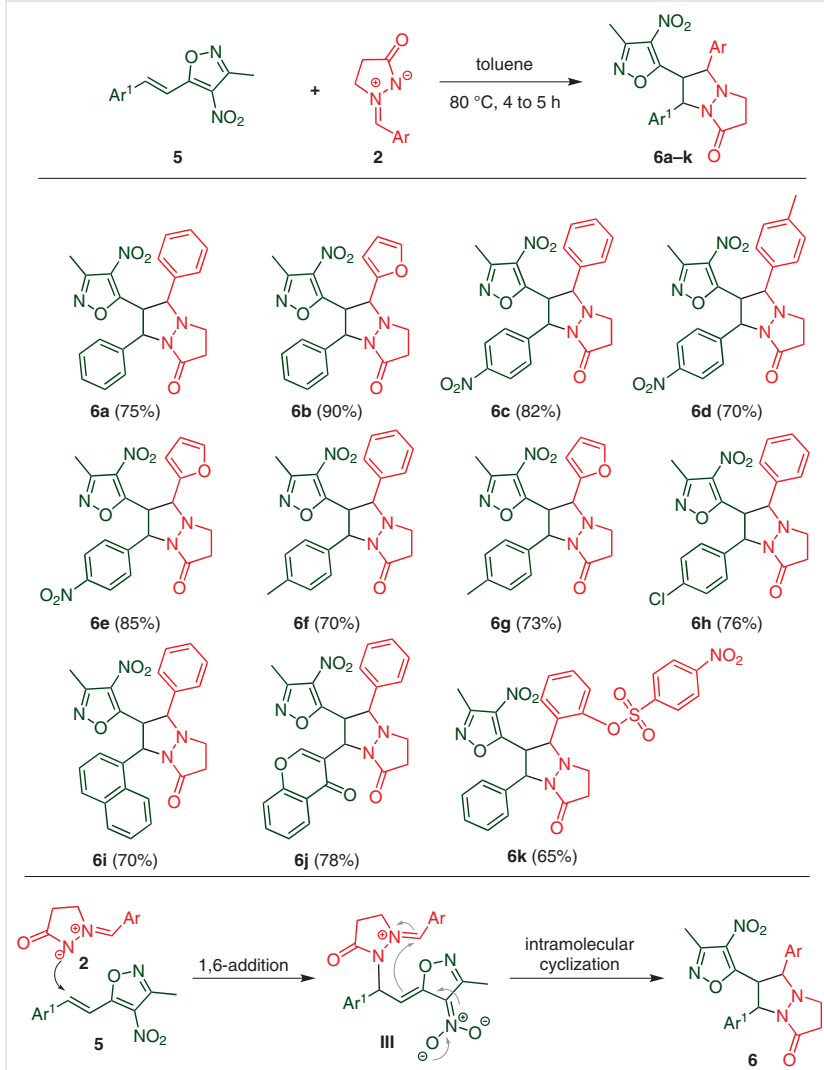

Scheme 6 Substrate scope for the synthesis of functionalized N,N'fused bicyclic pyrazolones and possible mechanism for the reaction

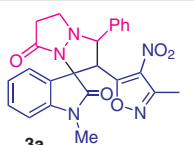

3a $\mathrm{Me}$

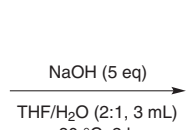

$80^{\circ} \mathrm{C}, 2 \mathrm{~h}$

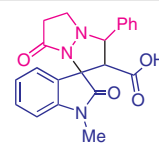

$7 a(78 \%)$
Scheme 7 Synthesis of spirooxindole-pyrazolone carboxylic acid derivative $7 a$

In summary, we have demonstrated a simple and catalyst-free [3+2]-cycloaddition reaction for the synthesis of dinitrogen-fused pyrazolone derivatives with moderate to good yields. The reaction proceeds via aza-1,4/1,6-Michael addition of azomethine imines onto a conjugated system followed by intramolecular cyclization. The generated dini-

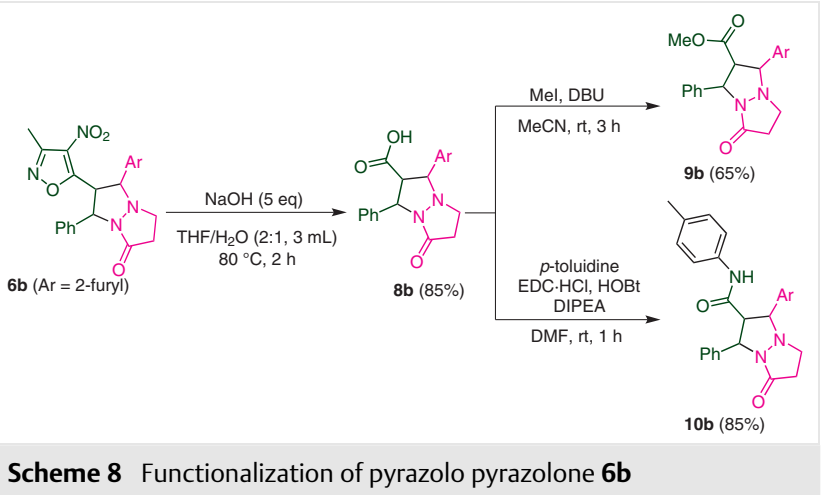

trogen-fused heterocyclic compounds $\mathbf{3 a}$ and $\mathbf{6 b}$ were converted into pyrazolone-based carboxylic acids by hydrolysis of the isoxazole ring. The carboxylic acid can be used as a starting point for the construction of hybrid molecules using esterification or amide bond formation, as shown in Scheme 8.

All the solvents and required chemicals were procured from SD-Fine, Sigma-Aldrich, and Spectrochem, and used without purification and distillation. ${ }^{1} \mathrm{H}$ and ${ }^{13} \mathrm{C}$ NMR spectra were recorded with Bruker Avance 400 or $500 \mathrm{MHz}$ spectrometers using $\mathrm{CDCl}_{3}$ or DMSO- $d_{6}$ as solvents and are reported in $\delta$ units (ppm). Mass spectra of all the compounds were record with an Agilent Technologies-6530 spectrometer.

\section{[3+2] Cycloaddition Reaction; General Procedure}

To a solution of isoxazole-styrene $\mathbf{1}$ or $\mathbf{5}(0.35 \mathrm{mmol}, 1$ equiv) in THF/toluene $(4 \mathrm{~mL})$ was added azomethine imine $2(0.35 \mathrm{mmol}, 1$ equiv) and the contents were heated at reflux (Table 1 ) for $4-6 \mathrm{~h}$. After completion of reaction (monitored by TLC) the mixture was cooled to r.t., solvent was evaporated, and the crude product was purified by silica gel column chromatography. Elution of the column with hexane/EtOAc (40-50\%) gave the desired products 3, 4, and $\mathbf{6}$.

\section{Synthesis of Pyrazolopyrazole Carboxylic Acids; General Proce- dure}

To a solution of cyclic adduct $3 \mathbf{a}$ or $\mathbf{6 b}(0.25 \mathrm{mmol}, 1$ equiv) in THF ( 2 $\mathrm{mL}$ ) was added aq. $\mathrm{NaOH}[1.25 \mathrm{mmol}, 5$ equiv] and the resulting mixture was heated at reflux for $2 \mathrm{~h}$. After completion of the reaction (monitored by TLC), the reaction was quenched with $2 \mathrm{M} \mathrm{HCl}$ at $0{ }^{\circ} \mathrm{C}$. The mixture was extracted with EtOAc $(3 \times 10 \mathrm{~mL})$, and the combined organic layers were dried using sodium sulfate, filtered, and evaporation of the solvent under reduced pressure gave the crude product, which was purified by silica gel column chromatography (hexane/ EtOAc) to give the desired products $\mathbf{7 a}$ or $\mathbf{8 b}$ as white solids.

Synthesis of Methyl 1-(Furan-2-yl)-5-oxo-3-phenylhexahydropyrazolo[1,2-a]pyrazole-2-carboxylate $(9 b)$

To a solution of carboxylic acid $\mathbf{8 b}(0.32 \mathrm{mmol}, 1$ equiv) $\mathrm{MeCN}(3 \mathrm{~mL})$ was added DBU ( $0.32 \mathrm{mmol}, 1$ equiv) and $\mathrm{MeI}(0.38 \mathrm{mmol}, 1.2$ equiv). The reaction mixture was stirred at r.t. for $3 \mathrm{~h}$, then the crude product was purified over silica gel by column chromatography to afford the desired product $\mathbf{9 b}$. 
Synthesis of 1-(Furan-2-yl)-5-oxo-3-phenyl- $N$-(p-tolyl)hexahydropyrazolo[1,2-a]pyrazole-2-carboxamide (10b)

To a solution of acid $\mathbf{8 b}(0.32 \mathrm{mmol}, 1$ equiv) in DMF ( $3 \mathrm{~mL})$ was added DIPEA ( $0.96 \mathrm{mmol}, 3$ equiv). The mixture was cooled to $0{ }^{\circ} \mathrm{C}$ and treated with $\mathrm{EDC} \cdot \mathrm{HCl}$ ( $0.64 \mathrm{mmol}, 2$ equiv), $\mathrm{HOBt}(0.64 \mathrm{mmol}, 2$ equiv) and the amine $(0.38 \mathrm{mmol}, 1.2$ equiv). The reaction mixture was then stirred at r.t. for $1 \mathrm{~h}$. After completion, the mixture was diluted with $\mathrm{H}_{2} \mathrm{O}(15 \mathrm{~mL})$ and extracted with EtOAc $(15 \mathrm{~mL})$. The organic layer was dried $\left(\mathrm{Na}_{2} \mathrm{SO}_{4}\right)$, filtered, and concentrated under reduced pressure. The resulting material was purified by silica gel column chromatography to provide the final product $\mathbf{1 0 b}$ as a white solid.

1-Methyl-2'-(3-Methyl-4-nitroisoxazol-5-yl)-3'-phenyl-5',6'-dihydro-2'H-spiro[indoline-3,1'-pyrazolo[1,2-a]pyrazole]-2,7'(3'H)-dione (3a)

Yield: $134 \mathrm{mg}$ (84\%); yellow solid; $\mathrm{mp} 183-185{ }^{\circ} \mathrm{C}$

${ }^{1} \mathrm{H}$ NMR $\left(400 \mathrm{MHz}, \mathrm{CDCl}_{3}\right): \delta=7.59(\mathrm{dd}, J=8.0,1.6 \mathrm{~Hz}, 2 \mathrm{H}), 7.34-7.27$ $(\mathrm{m}, 3 \mathrm{H}), 7.16(\mathrm{dd}, J=8.0,1.2 \mathrm{~Hz}, 1 \mathrm{H}), 7.04(\mathrm{dd}, J=7.2,0.8 \mathrm{~Hz}, 1 \mathrm{H})$, 6.89-6.82 (m, $1 \mathrm{H}), 6.69(\mathrm{~d}, J=8.0 \mathrm{~Hz}, 1 \mathrm{H}), 5.05(\mathrm{~d}, J=10.8 \mathrm{~Hz}, 1 \mathrm{H})$, $4.49(\mathrm{~d}, J=10.8 \mathrm{~Hz}, 1 \mathrm{H}), 3.55-3.48(\mathrm{~m}, 1 \mathrm{H}), 3.17(\mathrm{~s}, 3 \mathrm{H}), 2.99-2.80$ ( $\mathrm{m}, 2 \mathrm{H}), 2.64-2.57(\mathrm{~m}, 1 \mathrm{H}), 2.20(\mathrm{~s}, 3 \mathrm{H})$.

${ }^{13} \mathrm{C}$ NMR $\left(100 \mathrm{MHz}, \mathrm{CDCl}_{3}\right): \delta=172.89,167.35,163.10,155.70$, $144.12,133.83,131.04,130.76,129.43,129.18,128.19,124.08$, 122.73, 122.01, 108.98, 70.96, 64.18, 59.54, 51.09, 36.15, 26.95, 11.17. MS (ESI): $m / z$ calcd for $\mathrm{C}_{24} \mathrm{H}_{21} \mathrm{~N}_{5} \mathrm{O}_{5}: 459.1543$; found: 460.1614 [M + 1].

1-Ethyl-2'-(3-Methyl-4-nitroisoxazol-5-yl)-3'-phenyl-5',6'-dihydro-2' $H$-spiro[indoline-3,1'-pyrazolo[1,2-a]pyrazole]-2,7'(3'H)-dione (3b)

Yield: $120 \mathrm{mg}$ (75\%); white solid; $\mathrm{mp} 168-170{ }^{\circ} \mathrm{C}$.

${ }^{1} \mathrm{H}$ NMR $\left(400 \mathrm{MHz}, \mathrm{CDCl}_{3}\right): \delta=7.55(\mathrm{~d}, J=8.0 \mathrm{~Hz}, 2 \mathrm{H}), 7.27-7.14(\mathrm{~m}, 4$ H), $7.15(\mathrm{~d}, J=7.4 \mathrm{~Hz}, 1 \mathrm{H}), 6.94(\mathrm{t}, J=7.6 \mathrm{~Hz}, 1 \mathrm{H}), 6.80(\mathrm{~d}, J=8.0 \mathrm{~Hz}, 1$ H), $5.18(\mathrm{~d}, J=10.8 \mathrm{~Hz}, 1 \mathrm{H}), 4.56(\mathrm{~d}, J=10.8 \mathrm{~Hz}, 1 \mathrm{H}), 3.93-3.68(\mathrm{~m}, 2$ $\mathrm{H}), 3.58(\mathrm{t}, J=8.0 \mathrm{~Hz}, 1 \mathrm{H}), 2.97(\mathrm{~m} 2 \mathrm{H}), 2.73-2.62(\mathrm{~m}, 1 \mathrm{H}), 2.35(\mathrm{~s}, 3$ $\mathrm{H}), 1.30(\mathrm{t}, J=7.2 \mathrm{~Hz}, 3 \mathrm{H})$.

${ }^{13} \mathrm{C}$ NMR $\left(100 \mathrm{MHz}, \mathrm{CDCl}_{3}\right): \delta=172.03,166.93,163.18,155.75$, $142.05,133.57,131.12,130.70,129.51,129.23,128.12,127.96$, $124.85,123.98,109.97,70.88,63.94,59.06,51.09,36.13,35.74,12.01$, 11.23.

MS (ESI): $m / z$ calcd for $\mathrm{C}_{25} \mathrm{H}_{23} \mathrm{~N}_{5} \mathrm{O}_{5}$ : 473.1699; found: 474.1714 [M + 1].

2'-(3-Methyl-4-nitroisoxazol-5-yl)-3'-phenyl-1-propyl-5',6'-dihydro-2' H-spiro[indoline-3,1'-pyrazolo[1,2-a]pyrazole]-2,7'(3'H)-dione (3c)

Yield: $121 \mathrm{mg}$ (78\%); yellow solid; mp $168-170{ }^{\circ} \mathrm{C}$.

${ }^{1} \mathrm{H} \mathrm{NMR}\left(400 \mathrm{MHz}, \mathrm{CDCl}_{3}\right): \delta=7.67(\mathrm{~d}, J=6.5 \mathrm{~Hz}, 2 \mathrm{H}), 7.39(\mathrm{q}, J=6.4$ $\mathrm{Hz}, 3 \mathrm{H}), 7.24(\mathrm{~d}, J=7.8 \mathrm{~Hz}, 1 \mathrm{H}), 7.15(\mathrm{~d}, J=7.5 \mathrm{~Hz}, 1 \mathrm{H}), 6.93(\mathrm{t}, J=7.5$ $\mathrm{Hz}, 1 \mathrm{H}), 6.79(\mathrm{~d}, J=7.8 \mathrm{~Hz}, 1 \mathrm{H}), 5.19(\mathrm{~d}, J=10.7 \mathrm{~Hz}, 1 \mathrm{H}), 4.59(\mathrm{~d}, J=$ $10.7 \mathrm{~Hz}, 1 \mathrm{H}), 3.82-3.55(\mathrm{~m}, 3 \mathrm{H}), 2.99(\mathrm{dtd}, J=28.6,13.1,8.3 \mathrm{~Hz}, 2 \mathrm{H})$, 2.68 (dd, $J=15.1,6.6 \mathrm{~Hz}, 1 \mathrm{H}$ ), 2.30 (s, $3 \mathrm{H}$ ), 1.74 (td, $J=14.1,6.9 \mathrm{~Hz}, 2$ $\mathrm{H}), 1.00(\mathrm{t}, J=7.4 \mathrm{~Hz}, 3 \mathrm{H})$.

${ }^{13} \mathrm{C}$ NMR $\left(125 \mathrm{MHz}, \mathrm{CDCl}_{3}\right): \delta=172.81,167.41,162.99,155.71$, $143.93,133.92,130.68,129.40,129.17,128.16,127.41,124.31$, $122.47,122.18,109.21,71.00,64.15,59.18,51.12,42.49,36.23,20.42$, $11.35,11.22$

MS (ESI): $m / z$ calcd for $\mathrm{C}_{26} \mathrm{H}_{25} \mathrm{~N}_{5} \mathrm{O}_{5}$ : 487.1856; found: 488.1971 [M + 1] .
1-Benzyl-2'-(3-methyl-4-nitroisoxazol-5-yl)-3'-phenyl-5',6'-dihydro-2' $H$-spiro[indoline-3,1'-pyrazolo[1,2-a]pyrazole]-2,7'(3'H)-dione (3d)

Yield: $123 \mathrm{mg}$ (83\%); white solid; $\mathrm{mp} 175-177^{\circ} \mathrm{C}$.

${ }^{1} \mathrm{H}$ NMR $\left(400 \mathrm{MHz}, \mathrm{CDCl}_{3}\right): \delta=7.62-7.56(\mathrm{~m}, 2 \mathrm{H}), 7.35-7.23(\mathrm{~m}, 8 \mathrm{H})$, 7.09 (dd, $J=7.2,0.8 \mathrm{~Hz}, 1 \mathrm{H}$ ), 7.03 (td, $J=8.0,1.2 \mathrm{~Hz}, 1 \mathrm{H}), 6.83$ (td, $J=$ 7.6, $0.8 \mathrm{~Hz}, 1 \mathrm{H}), 6.48$ (d, J = 8.0 Hz, $1 \mathrm{H}), 5.23(\mathrm{~d}, J=16.0 \mathrm{~Hz}, 1 \mathrm{H}), 5.17$ $(\mathrm{d}, J=10.8 \mathrm{~Hz}, 1 \mathrm{H}), 4.59(\mathrm{~s}, 1 \mathrm{H}), 4.53(\mathrm{~d}, J=10.8 \mathrm{~Hz}, 1 \mathrm{H}), 3.52(\mathrm{t}, J=$ $8.4 \mathrm{~Hz}, 1 \mathrm{H}), 3.04-2.92(\mathrm{~m}, 1 \mathrm{H}), 2.91-2.82(\mathrm{~m}, 1 \mathrm{H}), 2.61$ (dd, $J=16.8$, $7.6 \mathrm{~Hz}, 1 \mathrm{H}), 2.21(\mathrm{~s}, 3 \mathrm{H})$

${ }^{13} \mathrm{C}$ NMR $\left(100 \mathrm{MHz}, \mathrm{CDCl}_{3}\right): \delta=173.07,167.30,163.14,155.72$, $143.48,134.71,133.78,131.09,130.68,129.46,129.20,128.86$, 128.17, 127.57, 127.04, 124.19, 122.81, 122.17, 110.18, 70.93, 64.13, 59.33, 51.19, 44.66, 36.29, 11.23 .

MS (ESI): $m / z$ calcd for $\mathrm{C}_{30} \mathrm{H}_{25} \mathrm{~N}_{5} \mathrm{O}_{5}: 535.1856$; found: 536.1940 [M + 1].

1-Methyl-2'-(3-methyl-4-nitroisoxazol-5-yl)-3'-(p-tolyl)-5',6'-dihydro-2' $H$-spiro[indoline-3,1'-pyrazolo[1,2-a]pyrazole]-2,7'(3'H)dione (3e)

Yield: $126 \mathrm{mg}$ (76\%); light-yellow solid; mp 189-191 ${ }^{\circ} \mathrm{C}$.

${ }^{1} \mathrm{H} \mathrm{NMR}\left(400 \mathrm{MHz}, \mathrm{CDCl}_{3}\right): \delta=7.56(\mathrm{~d}, J=8.0 \mathrm{~Hz}, 2 \mathrm{H}), 7.27-7.19(\mathrm{~m}, 3$ H), $7.13(\mathrm{~d}, J=7.6 \mathrm{~Hz}, 1 \mathrm{H}), 6.95(\mathrm{t}, J=7.6 \mathrm{~Hz}, 1 \mathrm{H}), 6.77(\mathrm{~d}, J=7.6 \mathrm{~Hz}, 1$ $\mathrm{H}), 5.13(\mathrm{~d}, J=10.4 \mathrm{~Hz}, 1 \mathrm{H}), 4.56(\mathrm{~d}, J=10.4 \mathrm{~Hz}, 1 \mathrm{H}), 3.59(\mathrm{t}, J=7.2$ $\mathrm{Hz}, 1 \mathrm{H}), 3.26$ (s, $3 \mathrm{H}), 3.09-2.87(\mathrm{~m}, 2 \mathrm{H}), 2.69$ (dd, J = 14.4, $6.4 \mathrm{~Hz}, 1$ $\mathrm{H}), 2.35$ (s, $3 \mathrm{H}), 2.29(\mathrm{~s}, 3 \mathrm{H})$.

${ }^{13} \mathrm{C}$ NMR $\left(100 \mathrm{MHz}, \mathrm{CDCl}_{3}\right): \delta=172.91,167.41,163.09,155.65$, $144.10,139.44,131.07,130.72,130.67,129.85,128.08,124.09$, $122.71,122.07,108.95,70.85,64.16,59.49,51.06,36.15,26.94,21.20$, 11.17.

MS (ESI): $m / z$ calcd for $\mathrm{C}_{25} \mathrm{H}_{23} \mathrm{~N}_{5} \mathrm{O}_{5}: 473.1699$; found: 474.2758 [M + 1].

1-Ethyl-2'-(3-methyl-4-nitroisoxazol-5-yl)-3'-(p-tolyl)-5',6'-dihydro-2' $H$-spiro[indoline-3,1'-pyrazolo[1,2-a]pyrazole]-2,7'(3'H)-dione (3f)

Yield: $114 \mathrm{mg}$ (70\%); white solid; $\mathrm{mp} 194-196{ }^{\circ} \mathrm{C}$.

${ }^{1} \mathrm{H}$ NMR $\left(400 \mathrm{MHz}, \mathrm{CDCl}_{3}\right): \delta=7.55(\mathrm{~d}, J=8.0 \mathrm{~Hz}, 2 \mathrm{H}), 7.26-7.18(\mathrm{~m}, 3$ $\mathrm{H}), 7.15(\mathrm{~d}, J=7.2 \mathrm{~Hz}, 1 \mathrm{H}), 6.94(\mathrm{t}, J=7.6 \mathrm{~Hz}, 1 \mathrm{H}), 6.80(\mathrm{~d}, J=8.0 \mathrm{~Hz}, 1$ H), $5.18(\mathrm{~d}, J=10.8 \mathrm{~Hz}, 1 \mathrm{H}), 4.56(\mathrm{~d}, J=10.8 \mathrm{~Hz}, 1 \mathrm{H}), 3.93-3.68(\mathrm{~m}, 2$ $\mathrm{H}), 3.58(\mathrm{t}, J=8.0 \mathrm{~Hz}, 1 \mathrm{H}), 3.10-2.84(\mathrm{~m}, 2 \mathrm{H}), 2.73-2.62(\mathrm{~m}, 1 \mathrm{H}), 2.35$ (s, $3 \mathrm{H}), 2.30(\mathrm{~s}, 3 \mathrm{H}), 1.30$ (t, $J=7.2 \mathrm{~Hz}, 3 \mathrm{H})$.

${ }^{13} \mathrm{C}$ NMR $\left(100 \mathrm{MHz}, \mathrm{CDCl}_{3}\right): \delta=172.43,167.42,163.04,155.70$, $143.43,133.90,131.00,130.72,129.41,129.18,128.17,124.37$, $122.50,122.27,109.05,71.00,64.26,59.13,51.08,36.19,35.58,12.08$, 11.21.

MS (ESI): $m / z$ calcd for $\mathrm{C}_{26} \mathrm{H}_{25} \mathrm{~N}_{5} \mathrm{O}_{5}$ : 487.1856; found: 488.1868 [M + 1].

1-Benzyl-3'-(4-methoxyphenyl)-2'-(3-methyl-4-nitroisoxazol-5yl)-5',6'-dihydro-2' $H$-spiro[indoline-3,1'-pyrazolo[1,2-a]pyrazole]2,7'(3'H)-dione (3g)

Yield: $109 \mathrm{mg}(70 \%) ;$ white solid; $\mathrm{mp} 175-177^{\circ} \mathrm{C}$.

${ }^{1} \mathrm{H}$ NMR (400 MHz, $\mathrm{CDCl}_{3}$ ): $\delta=7.51(\mathrm{~d}, J=8.6 \mathrm{~Hz}, 2 \mathrm{H}), 7.25(\mathrm{~m}, 5 \mathrm{H})$, $7.10(\mathrm{~d}, J=7.4 \mathrm{~Hz}, 1 \mathrm{H}), 7.02(\mathrm{t}, J=7.5 \mathrm{~Hz}, 1 \mathrm{H}), 6.83(\mathrm{t}, J=6.7 \mathrm{~Hz}, 3 \mathrm{H})$, $6.47(\mathrm{~d}, J=7.8 \mathrm{~Hz}, 1 \mathrm{H}), 5.22(\mathrm{~d}, J=16.1 \mathrm{~Hz}, 1 \mathrm{H}), 5.14(\mathrm{~d}, J=10.7 \mathrm{~Hz}, 1$ $\mathrm{H}), 4.57(\mathrm{~d}, J=16.1 \mathrm{~Hz}, 1 \mathrm{H}), 4.50(\mathrm{~d}, J=10.7 \mathrm{~Hz}, 1 \mathrm{H}), 3.50(\mathrm{t}, J=8.1$ $\mathrm{Hz}, 1 \mathrm{H}), 3.40(\mathrm{~s}, 3 \mathrm{H}), 3.02-2.81(\mathrm{~m}, 2 \mathrm{H}), 2.61(\mathrm{dd}, J=15.4,7.1 \mathrm{~Hz}, 1$ $\mathrm{H}), 2.21(\mathrm{~s}, 3 \mathrm{H})$. 
${ }^{13} \mathrm{C}$ NMR $\left(125 \mathrm{MHz}, \mathrm{CDCl}_{3}\right): \delta=173.10,167.39,163.14,160.45$, $155.69,143.43,134.72,131.13,130.65,129.37,128.86,127.57$, $127.03,125.34,124.20,122.81,122.25,114.58,110.16,70.58,64.10$, $59.24,55.33,51.16,44.65,36.27,11.23$.

MS (ESI): $m / z$ calcd for $\mathrm{C}_{31} \mathrm{H}_{27} \mathrm{~N}_{5} \mathrm{O}_{6}: 565.1961$; found: 566.2041 [M + 1].

5-Chloro-1-methyl-2'-(3-methyl-4-nitroisoxazol-5-yl)-3'-phenyl5',6'-dihydro-2'H-spiro[indoline-3,1'-pyrazolo[1,2-a]pyrazole]2,7'(3'H)-dione (3h)

Yield: $122 \mathrm{mg}$ (79\%); white solid; $\mathrm{mp} 166-168{ }^{\circ} \mathrm{C}$.

${ }^{1} \mathrm{H}$ NMR $\left(400 \mathrm{MHz}, \mathrm{CDCl}_{3}\right): \delta=7.64(\mathrm{~d}, J=6.4 \mathrm{~Hz}, 2 \mathrm{H}), 7.41-7.33(\mathrm{~m}, 3$ $\mathrm{H}), 7.23(\mathrm{dd}, J=8.0,1.6 \mathrm{~Hz}, 1 \mathrm{H}), 7.10(\mathrm{~d}, J=1.6 \mathrm{~Hz}, 1 \mathrm{H}), 6.69(\mathrm{~d}, J=8.0$ $\mathrm{Hz}, 1 \mathrm{H}), 5.11(\mathrm{~d}, J=10.8 \mathrm{~Hz}, 1 \mathrm{H}), 4.55(\mathrm{~d}, J=10.8 \mathrm{~Hz}, 1 \mathrm{H}), 3.58(\mathrm{t}, J=$ 7.6 Hz, $1 \mathrm{H}), 3.23$ (s, $3 \mathrm{H}), 3.07-2.89(\mathrm{~m}, 2 \mathrm{H}), 2.73-2.65(\mathrm{~m}, 1 \mathrm{H}), 2.32$ (

${ }^{13} \mathrm{C}$ NMR $\left(100 \mathrm{MHz}, \mathrm{CDCl}_{3}\right): \delta=172.49,166.88,163.23,155.75$, $142.76,133.50,131.18,130.72,129.53,129.24,128.21,128.15$, 124.59, 123.70, 109.91, 70.86, 63.88, 59.46, 51.11, 36.11, 27.08, 11.20. MS (ESI): $m / z$ calcd for $\mathrm{C}_{24} \mathrm{H}_{20} \mathrm{ClN}_{5} \mathrm{O}_{5}: 493.1153$; found: 494.1235 [M+1].

5-Chloro-1-methyl-2'-(3-methyl-4-nitroisoxazol-5-yl)-3'-(p-tolyl)5',6'-dihydro-2' $H$-spiro[indoline-3,1'-pyrazolo[1,2-a]pyrazole]2,7'(3'H)-dione (3i)

Yield: $127 \mathrm{mg}$ (80\%); white solid; $\mathrm{mp} 223-225^{\circ} \mathrm{C}$.

${ }^{1} \mathrm{H}$ NMR $\left(400 \mathrm{MHz}, \mathrm{CDCl}_{3}\right): \delta=7.54(\mathrm{~d}, J=8.0 \mathrm{~Hz}, 2 \mathrm{H}), 7.25(\mathrm{dd}, J=8.4$, $2.0 \mathrm{~Hz}, 1 \mathrm{H}), 7.21(\mathrm{~d}, J=8.0 \mathrm{~Hz}, 2 \mathrm{H}), 7.12(\mathrm{~d}, J=2.0 \mathrm{~Hz}, 1 \mathrm{H}), 6.71(\mathrm{~d}, J=$ $8.4 \mathrm{~Hz}, 1 \mathrm{H}), 5.11$ (d, $J=10.8 \mathrm{~Hz}, 1 \mathrm{H}), 4.54(\mathrm{~d}, J=10.8 \mathrm{~Hz}, 1 \mathrm{H}), 3.62-$ $3.56(\mathrm{~m}, 1 \mathrm{H}), 3.25(\mathrm{~s}, 3 \mathrm{H}), 3.08-2.88(\mathrm{~m}, 2 \mathrm{H}), 2.74-2.66(\mathrm{~m}, 1 \mathrm{H})$, 2.35 (s, $3 \mathrm{H}), 2.33$ (s, $3 \mathrm{H})$.

${ }^{13} \mathrm{C}$ NMR $\left(100 \mathrm{MHz}, \mathrm{CDCl}_{3}\right): \delta=172.03,166.93,163.18,155.75$, $142.05,133.57,131.12,130.70,129.51,129.23,128.12,127.96$, 124.85, 123.98, 109.97, 70.88, 63.94, 59.06, 51.09, 36.13, 35.74, 12.01, 11.23 .

MS (ESI): $m / z$ calcd for $\mathrm{C}_{25} \mathrm{H}_{22} \mathrm{ClN}_{5} \mathrm{O}_{5}$ : 507.1309; found: 508.1412 [M + 1].

5-Chloro-3'-(4-methoxyphenyl)-1-methyl-2'-(3-methyl-4-nitroisoxazol-5-yl)-5',6'-dihydro-2' $\mathrm{H}$-spiro[indoline-3,1'-pyrazolo[1,2a]pyrazole]-2,7'(3'H)-dione ( $3 \mathbf{j})$

Yield: $131 \mathrm{mg}$ (80\%); light-yellow solid; mp 194-196 ${ }^{\circ} \mathrm{C}$.

${ }^{1} \mathrm{H}$ NMR $\left(400 \mathrm{MHz}, \mathrm{CDCl}_{3}\right): \delta=7.58(\mathrm{~d}, J=8.8 \mathrm{~Hz}, 2 \mathrm{H}), 7.25$ (dd, $J=8.4$, $2.0 \mathrm{~Hz}, 1 \mathrm{H}), 7.12(\mathrm{~d}, J=1.6 \mathrm{~Hz}, 1 \mathrm{H}), 6.92(\mathrm{~d}, J=8.8 \mathrm{~Hz}, 2 \mathrm{H}), 6.71(\mathrm{~d}, J$ $=8.0 \mathrm{~Hz}, 1 \mathrm{H}), 5.09(\mathrm{~d}, J=10.8 \mathrm{~Hz}, 1 \mathrm{H}), 4.52(\mathrm{~d}, J=10.8 \mathrm{~Hz}, 1 \mathrm{H}), 3.81$ (s, $3 \mathrm{H}), 3.63-3.54(\mathrm{~m}, 1 \mathrm{H}), 3.25$ (s, $3 \mathrm{H}), 3.08-2.87$ (m, $2 \mathrm{H}), 2.74-$ $2.66(\mathrm{~m}, 1 \mathrm{H}), 2.34(\mathrm{~s}, 3 \mathrm{H})$.

${ }^{13} \mathrm{C}$ NMR $\left(100 \mathrm{MHz}, \mathrm{CDCl}_{3}\right): \delta=172.50,166.98,163.20,160.50$, $155.70,142.72,131.22,130.67,129.35,128.19,125.06,124.58$, 123.78, 114.60, 109.86, 70.51, 63.84, 59.37, 55.33, 51.06, 36.08, 27.07, 11.20 .

MS (ESI): $m / z$ calcd for $\mathrm{C}_{25} \mathrm{H}_{22} \mathrm{ClN}_{5} \mathrm{O}_{6}: 523.1259$; found: 524.1379 [M+1].

5-Chloro-1-ethyl-2'-(3-methyl-4-nitroisoxazol-5-yl)-3'-phenyl5',6'-dihydro-2'H-spiro[indoline-3,1'-pyrazolo[1,2-a]pyrazole]2,7'(3'H)-dione (3k)

Yield: $114 \mathrm{mg}$ (75\%); light-yellow solid; mp 193-195 ${ }^{\circ} \mathrm{C}$.
${ }^{1} \mathrm{H}$ NMR $\left(400 \mathrm{MHz}, \mathrm{CDCl}_{3}\right): \delta=7.57(\mathrm{dd}, J=7.6,1.6 \mathrm{~Hz}, 2 \mathrm{H}), 7.34-7.27$ ( $\mathrm{m}, 3 \mathrm{H}), 7.15(\mathrm{dd}, J=8.4,2.0 \mathrm{~Hz}, 1 \mathrm{H}), 7.04(\mathrm{~d}, J=2.0 \mathrm{~Hz}, 1 \mathrm{H}), 6.64(\mathrm{~d}$, $J=8.4 \mathrm{~Hz}, 1 \mathrm{H}), 5.09(\mathrm{~d}, J=10.4 \mathrm{~Hz}, 1 \mathrm{H}), 4.48(\mathrm{~d}, J=10.4 \mathrm{~Hz}, 1 \mathrm{H})$, 3.81-3.70 (m, $1 \mathrm{H}), 3.70-3.58(\mathrm{~m}, 1 \mathrm{H}), 3.53-3.46(\mathrm{~m}, 1 \mathrm{H}), 2.99-2.80$ (m, $2 \mathrm{H}), 2.64-2.56(\mathrm{~m}, 1 \mathrm{H}), 2.25(\mathrm{~s}, 3 \mathrm{H}), 1.20(\mathrm{~d}, J=7.2 \mathrm{~Hz}, 3 \mathrm{H})$.

${ }^{13} \mathrm{C}$ NMR $\left(100 \mathrm{MHz}, \mathrm{CDCl}_{3}\right): \delta=172.03,166.93,163.18,155.75$, $142.05,133.57,131.12,130.70,129.51,129.23,128.12,127.96$, 124.85, 123.98, 109.97, 70.88, 63.94, 59.06, 51.09, 36.13, 35.74, 12.01, 11.23.

MS (ESI): $m / z$ calcd for $\mathrm{C}_{25} \mathrm{H}_{22} \mathrm{ClN}_{5} \mathrm{O}_{5}$ : 507.1309; found: 508.1393 [M + 1].

3'-(Furan-2-yl)-2'-(3-methyl-4-nitroisoxazol-5-yl)-1-propyl-5',6'dihydro-2'H-spiro[indoline-3,1'-pyrazolo[1,2-a]pyrazole]2,7'(3'H)-dione (31)

Yield: $109 \mathrm{mg}$ (72\%); white solid; $\mathrm{mp} 180-183{ }^{\circ} \mathrm{C}$.

${ }^{1} \mathrm{H} \mathrm{NMR}\left(400 \mathrm{MHz}, \mathrm{CDCl}_{3}\right): \delta=7.45(\mathrm{~d}, J=0.8 \mathrm{~Hz}, 1 \mathrm{H}), 7.24(\mathrm{t}, J=7.6$ $\mathrm{Hz}, 1 \mathrm{H}), 7.09(\mathrm{~d}, J=7.6 \mathrm{~Hz}, 1 \mathrm{H}), 6.91(\mathrm{t}, J=7.6 \mathrm{~Hz}, 1 \mathrm{H}), 6.79(\mathrm{~d}, J=8.0$ $\mathrm{Hz}, 1 \mathrm{H}), 6.59$ (s, $1 \mathrm{H}), 6.39-6.35(\mathrm{~m}, 1 \mathrm{H}), 5.47(\mathrm{~d}, J=10.4 \mathrm{~Hz}, 1 \mathrm{H})$, $4.77(\mathrm{~d}, J=10.4 \mathrm{~Hz}, 1 \mathrm{H}), 3.81-3.69(\mathrm{~m}, 2 \mathrm{H}), 3.68-3.60(\mathrm{~m}, 1 \mathrm{H}), 3.11-$ $2.91(\mathrm{~m}, 2 \mathrm{H}), 2.72-2.61(\mathrm{~m}, 1 \mathrm{H}), 2.34(\mathrm{~s}, 3 \mathrm{H}), 1.82-1.70(\mathrm{~m}, 2 \mathrm{H})$, $1.00(\mathrm{t}, J=7.6 \mathrm{~Hz}, 3 \mathrm{H})$.

${ }^{13} \mathrm{C}$ NMR $\left(100 \mathrm{MHz}, \mathrm{CDCl}_{3}\right): \delta=172.35,167.17,163.06,155.69$, $146.68,143.99,143.88,130.76,124.23,122.44,122.09,110.73$, $110.15,109.21,63.98,63.89,55.07,51.43,42.47,36.00,20.40,11.32$, 11.23.

MS (ESI): $m / z$ calcd for $\mathrm{C}_{24} \mathrm{H}_{23} \mathrm{~N}_{5} \mathrm{O}_{6}: 477.1648$; found: 478.1706 [M + 1].

5-Chloro-1-ethyl-3'-(furan-2-yl)-2'-(3-methyl-4-nitroisoxazol-5yl)-5',6'-dihydro-2' $H$-spiro[indoline-3,1'-pyrazolo[1,2-a]pyrazole]2,7'(3'H)-dione (3m)

Yield: $111 \mathrm{mg}$ (75\%); white solid; $\mathrm{mp} 170-172{ }^{\circ} \mathrm{C}$.

${ }^{1} \mathrm{H} \mathrm{NMR}\left(400 \mathrm{MHz}, \mathrm{CDCl}_{3}\right): \delta=7.46(\mathrm{~d}, J=0.8 \mathrm{~Hz}, 1 \mathrm{H}), 7.24(\mathrm{dd}, J=8.4$, $2.0 \mathrm{~Hz}, 1 \mathrm{H}), 7.08(\mathrm{~d}, J=2.0 \mathrm{~Hz}, 1 \mathrm{H}), 6.74(\mathrm{~d}, J=8.4 \mathrm{~Hz}, 1 \mathrm{H}), 6.59(\mathrm{~s}, 1$ H), $6.41-6.36(\mathrm{~m}, 1 \mathrm{H}), 5.46(\mathrm{~d}, J=10.4 \mathrm{~Hz}, 1 \mathrm{H}), 4.75(\mathrm{~d}, J=10.4 \mathrm{~Hz}, 1$ H), 3.90-3.79 (m, $1 \mathrm{H}), 3.79-3.65(\mathrm{~m}, 2 \mathrm{H}), 3.14-2.91(\mathrm{~m}, 2 \mathrm{H}), 2.69$ (dd, $J=16.0,6.8 \mathrm{~Hz}, 1 \mathrm{H}), 2.39$ (s, $3 \mathrm{H}), 1.29(\mathrm{t}, J=7.2 \mathrm{~Hz}, 3 \mathrm{H}$ ).

${ }^{13} \mathrm{C}$ NMR $\left(100 \mathrm{MHz}, \mathrm{CDCl}_{3}\right): \delta=171.72,166.81,163.29,155.75$, $146.42,144.00,142.11,131.03,130.78,127.95,124.77,123.90$, 110.77, 110.40, 109.99, 63.84, 63.70, 54.95, 51.32, 35.90, 35.74, 12.00, 11.24

MS (ESI): $m / z$ calcd for $\mathrm{C}_{23} \mathrm{H}_{20} \mathrm{ClN}_{5} \mathrm{O}_{6}: 497.1102$; found: 498.1188 [M+1].

1-Methyl-3'-(3-methyl-4-nitroisoxazol-5-yl)-1'-phenyl-6',7'-dihydro-1'H-spiro[indoline-3,2'-pyrazolo[1,2-a]pyrazole]-2,5'(3'H)-dione (4a)

Yield: $120 \mathrm{mg}$ (75\%); white solid; $\mathrm{mp} 161-162{ }^{\circ} \mathrm{C}$.

${ }^{1} \mathrm{H}$ NMR $\left(400 \mathrm{MHz}, \mathrm{CDCl}_{3}\right): \delta=7.22(\mathrm{t}, J=7.6 \mathrm{~Hz}, 2 \mathrm{H}), 7.14(\mathrm{t}, J=7.6$ $\mathrm{Hz}, 2 \mathrm{H}), 6.99(\mathrm{~d}, J=7.6 \mathrm{~Hz}, 2 \mathrm{H}), 6.89(\mathrm{t}, J=7.6 \mathrm{~Hz}, 1 \mathrm{H}), 6.57(\mathrm{~d}, J=8.0$ $\mathrm{Hz}, 1 \mathrm{H}), 6.47(\mathrm{~d}, J=31.2 \mathrm{~Hz}, 1 \mathrm{H}), 5.97(\mathrm{~s}, 1 \mathrm{H}), 4.12(\mathrm{~s}, 1 \mathrm{H}), 3.69(\mathrm{t}, J=$ 8.4 Hz, $1 \mathrm{H}), 3.36-3.15$ (m, $2 \mathrm{H}), 3.02-2.93$ (m, $1 \mathrm{H}), 2.84$ (s, $3 \mathrm{H}), 2.49$ $(\mathrm{s}, 3 \mathrm{H})$.

${ }^{13} \mathrm{C}$ NMR $\left(125 \mathrm{MHz}, \mathrm{CDCl}_{3}\right): \delta=173.54,167.82,164.29,156.16$, $144.28,130.35,129.09,128.30,128.07,127.59,127.32,123.01$, 122.87, 122.48, 108.69, 77.28, 67.59, 54.07, 52.27, 36.63, 26.19, 11.31. MS (ESI): $m / z$ calcd for $\mathrm{C}_{24} \mathrm{H}_{21} \mathrm{~N}_{5} \mathrm{O}_{5}: 459.1543$; found: 460.1591 [M + 1]. 
1-Ethyl-3'-(3-methyl-4-nitroisoxazol-5-yl)-1'-phenyl-6',7'-dihydro-1' $H$-spiro[indoline-3,2'-pyrazolo[1,2-a]pyrazole]-2,5'(3'H)-dione (4b)

Yield: $128 \mathrm{mg}(81 \%)$; white solid; $\mathrm{mp} 156-158{ }^{\circ} \mathrm{C}$.

${ }^{1} \mathrm{H}$ NMR $\left(400 \mathrm{MHz}, \mathrm{CDCl}_{3}\right): \delta=7.24(\mathrm{t}, J=7.6 \mathrm{~Hz}, 2 \mathrm{H}), 7.16(\mathrm{t}, J=7.2$ $\mathrm{Hz}, 2 \mathrm{H}), 7.00$ (d, $J=7.6 \mathrm{~Hz}, 2 \mathrm{H}), 6.90(\mathrm{t}, J=7.6 \mathrm{~Hz}, 1 \mathrm{H}), 6.60(\mathrm{~d}, J=8.0$ $\mathrm{Hz}, 1 \mathrm{H}), 6.50(\mathrm{~d}, J=7.6 \mathrm{~Hz}, 1 \mathrm{H}), 5.99(\mathrm{~s}, 1 \mathrm{H}), 4.15(\mathrm{~s}, 1 \mathrm{H}), 3.73(\mathrm{t}, J=$ $8.4 \mathrm{~Hz}, 1 \mathrm{H}), 3.67-3.55(\mathrm{~m}, 1 \mathrm{H}), 3.34-3.19(\mathrm{~m}, 2 \mathrm{H}), 3.09-2.96(\mathrm{~m}, 1$ $\mathrm{H}), 2.92-2.82(\mathrm{~m}, 1 \mathrm{H}), 2.51(\mathrm{~s}, 3 \mathrm{H}), 0.68(\mathrm{t}, J=7.2 \mathrm{~Hz}, 3 \mathrm{H})$.

${ }^{13} \mathrm{C}$ NMR $\left(100 \mathrm{MHz}, \mathrm{CDCl}_{3}\right): \delta=173.07,167.83,164.22,156.10$, $143.43,130.40,130.27,129.27,129.02,128.28,127.34,123.23$, $123.13,122.24,108.73,77.42,67.46,53.91,52.20,36.67,34.53,11.61$, 11.31.

MS (ESI): $m / z$ calcd for $\mathrm{C}_{25} \mathrm{H}_{23} \mathrm{~N}_{5} \mathrm{O}_{5}$ : 473.1699; found: 474.1714 [M + 1].

\section{1-Benzyl-3'-(3-methyl-4-nitroisoxazol-5-yl)-1'-phenyl-6',7'-dihy- dro-1' $\mathrm{H}$-spiro[indoline-3,2'-pyrazolo[1,2-a]pyrazole]-2,5'(3'H)-di- one (4c)}

Yield: $125 \mathrm{mg}$ (85\%); light-yellow solid; mp $160-162{ }^{\circ} \mathrm{C}$.

${ }^{1} \mathrm{H}$ NMR $\left(400 \mathrm{MHz}, \mathrm{CDCl}_{3}\right): \delta=7.36(\mathrm{t}, J=7.2 \mathrm{~Hz}, 1 \mathrm{H}), 7.22(\mathrm{t}, J=7.6$ $\mathrm{Hz}, 2 \mathrm{H}), 7.19-7.14(\mathrm{~m}, 1 \mathrm{H}), 7.14-7.06(\mathrm{~m}, 5 \mathrm{H}), 6.87(\mathrm{t}, J=7.6 \mathrm{~Hz}, 1$ H), $6.55(\mathrm{~d}, J=7.6 \mathrm{~Hz}, 1 \mathrm{H}), 6.44(\mathrm{~d}, J=7.2 \mathrm{~Hz}, 2 \mathrm{H}), 6.37(\mathrm{~d}, J=8.0 \mathrm{~Hz}$, $1 \mathrm{H}), 6.06(\mathrm{~s}, 1 \mathrm{H}), 5.09(\mathrm{~d}, J=16.4 \mathrm{~Hz}, 1 \mathrm{H}), 4.33(\mathrm{~d}, J=16.4 \mathrm{~Hz}, 1 \mathrm{H})$, $4.26(\mathrm{~s}, 1 \mathrm{H}), 3.76(\mathrm{t}, J=8.4 \mathrm{~Hz}, 1 \mathrm{H}), 3.35-3.22(\mathrm{~m}, 1 \mathrm{H}), 3.08-2.98(\mathrm{~m}$, $1 \mathrm{H}), 2.89$ (dd, $J=16.0,7.6 \mathrm{~Hz}, 1 \mathrm{H}), 2.51(\mathrm{~s}, 3 \mathrm{H})$.

${ }^{13} \mathrm{C}$ NMR $\left(100 \mathrm{MHz}, \mathrm{CDCl}_{3}\right): \delta=173.75,167.69,164.29,156.10$, $143.84,143.53,134.55,130.58,130.33,129.17,128.71,128.63$, 127.70, 127.24, 126.29, 123.09, 122.81, 122.46, 110.03, 77.24, 67.54, 54.59, 52.29, 43.91, 36.66, 11.29 .

MS (ESI): $m / z$ calcd for $\mathrm{C}_{30} \mathrm{H}_{25} \mathrm{~N}_{5} \mathrm{O}_{5}$ : 535.1856; found: 536.1940 [M + 1].

3'-(3-Methyl-4-nitroisoxazol-5-yl)-1'-phenyl-1-propyl-6',7'-dihydro-1'H-spiro[indoline-3,2'-pyrazolo[1,2-a]pyrazole]-2,5'(3'H)-dione (4d)

Yield: $132 \mathrm{mg}$ (85\%); yellow solid; $\mathrm{mp} 155-157^{\circ} \mathrm{C}$.

${ }^{1} \mathrm{H} \mathrm{NMR}\left(400 \mathrm{MHz}, \mathrm{CDCl}_{3}\right): \delta=7.24-7.18(\mathrm{~m}, 2 \mathrm{H}), 7.14(\mathrm{t}, J=7.6 \mathrm{~Hz}, 2$ $\mathrm{H}), 7.00(\mathrm{~d}, J=7.2 \mathrm{~Hz}, 2 \mathrm{H}), 6.86(\mathrm{t}, J=7.6 \mathrm{~Hz}, 1 \mathrm{H}), 6.58(\mathrm{~d}, J=8.0 \mathrm{~Hz}, 1$ H), $6.48(\mathrm{~d}, J=7.6 \mathrm{~Hz}, 1 \mathrm{H}), 5.96(\mathrm{~s}, 1 \mathrm{H}), 4.14(\mathrm{~s}, 1 \mathrm{H}), 3.70(\mathrm{t}, J=8.4 \mathrm{~Hz}$, $1 \mathrm{H}), 3.55-3.46(\mathrm{~m}, 1 \mathrm{H}), 3.31-3.13(\mathrm{~m}, 2 \mathrm{H}), 3.03-2.92(\mathrm{~m}, 1 \mathrm{H}), 2.85$ (m, $1 \mathrm{H}), 2.49(\mathrm{~s}, 3 \mathrm{H}), 1.22-1.07(\mathrm{~m}, 2 \mathrm{H}), 0.58(\mathrm{t}, J=7.6 \mathrm{~Hz}, 3 \mathrm{H})$.

${ }^{13} \mathrm{C}$ NMR $\left(125 \mathrm{MHz}, \mathrm{CDCl}_{3}\right): \delta=173.40,167.82,164.23,156.10$, $144.06,130.46,130.26,129.05,128.34,128.16,127.41,123.16$, $122.96,122.15,108.93,77.22,67.37,54.13,52.28,41.67,36.65,20.20$, $11.31,11.00$

MS (ESI): $m / z$ calcd for $\mathrm{C}_{26} \mathrm{H}_{25} \mathrm{~N}_{5} \mathrm{O}_{5}$ : 487.1856; found: 488.1971 [M + 1].

1-Allyl-3'-(3-methyl-4-nitroisoxazol-5-yl)-1'-phenyl-6',7'-dihydro-1'H-spiro[indoline-3,2'-pyrazolo[1,2-a]pyrazole]-2,5'(3'H)-dione (4e)

Yield: $134 \mathrm{mg}$ (86\%); white solid; mp $162-164{ }^{\circ} \mathrm{C}$.

${ }^{1} \mathrm{H}$ NMR (400 MHz, $\mathrm{CDCl}_{3}$ ): $\delta=7.27-7.12(\mathrm{~m}, 4 \mathrm{H}), 7.00(\mathrm{~d}, J=7.2 \mathrm{~Hz}, 2$ H), 6.93-6.87 (m, $1 \mathrm{H}), 6.53(\mathrm{dd}, J=27.2,7.6 \mathrm{~Hz}, 2 \mathrm{H}), 5.97(\mathrm{~s}, 1 \mathrm{H})$, 5.24-5.16 (m, $1 \mathrm{H}), 4.83(\mathrm{~d}, J=10.4 \mathrm{~Hz}, 1 \mathrm{H}), 4.32(\mathrm{dd}, J=60.4,17.6 \mathrm{~Hz}$, $2 \mathrm{H}), 4.16(\mathrm{~s}, 1 \mathrm{H}), 3.82(\mathrm{dd}, J=16.4,4.0 \mathrm{~Hz}, 1 \mathrm{H}), 3.70(\mathrm{t}, J=8.4 \mathrm{~Hz}, 1$ $\mathrm{H}), 3.30-3.17(\mathrm{~m}, 1 \mathrm{H}), 3.07-2.82(\mathrm{~m}, 2 \mathrm{H}), 2.49(\mathrm{~s}, 3 \mathrm{H})$.

MS (ESI): $m / z$ calcd for $\mathrm{C}_{26} \mathrm{H}_{23} \mathrm{~N}_{5} \mathrm{O}_{5}$ : 485.1699; found: 486.1767 [M + 1].
1-Ethyl-3'-(3-methyl-4-nitroisoxazol-5-yl)-1'-(p-tolyl)-6',7'-dihydro-1' $H$-spiro[indoline-3,2'-pyrazolo[1,2-a]pyrazole]-2,5'(3'H)-dione (4f)

Yield: $136 \mathrm{mg}$ (84\%); white solid; $\mathrm{mp} 203-205^{\circ} \mathrm{C}$.

${ }^{1} \mathrm{H} \mathrm{NMR}\left(400 \mathrm{MHz}, \mathrm{CDCl}_{3}\right): \delta=7.06(\mathrm{~d}, J=7.6 \mathrm{~Hz}, 1 \mathrm{H}), 7.01-6.89(\mathrm{~m}, 3$ $\mathrm{H}), 6.88(\mathrm{~d}, J=8.0 \mathrm{~Hz}, 2 \mathrm{H}), 6.70(\mathrm{t}, J=7.6 \mathrm{~Hz}, 1 \mathrm{H}), 6.49(\mathrm{~d}, J=8.0 \mathrm{~Hz}, 1$ $\mathrm{H}), 6.26(\mathrm{~s}, 1 \mathrm{H}), 4.35(\mathrm{~s}, 1 \mathrm{H}), 3.91-3.81(\mathrm{~m}, 1 \mathrm{H}), 3.79-3.69(\mathrm{~m}, 1 \mathrm{H})$, 3.71-3.63 (m, 1 H), 3.26-3.16 (m, $1 \mathrm{H}), 2.97-2.88(\mathrm{~m}, 2 \mathrm{H}), 2.35(\mathrm{~s}, 3$ $\mathrm{H}), 2.17(\mathrm{~s}, 3 \mathrm{H}), 1.20(\mathrm{t}, J=7.2 \mathrm{~Hz}, 3 \mathrm{H})$.

${ }^{13} \mathrm{C}$ NMR $\left(100 \mathrm{MHz}, \mathrm{CDCl}_{3}\right): \delta=174.50,169.61,160.76,157.62$, $155.55,142.53,138.26,129.20,128.74,128.56,127.28,126.08$, 124.30, 121.61, 108.23, 78.14, 66.01, 59.04, 45.91, 35.16, 30.34, 21.07, $12.15,11.11$.

MS (ESI): $m / z$ calcd for $\mathrm{C}_{26} \mathrm{H}_{25} \mathrm{~N}_{5} \mathrm{O}_{5}: 487.1856$; found: 488.1868 [M + 1].

1-Benzyl-3'-(3-methyl-4-nitroisoxazol-5-yl)-1'-(p-tolyl)-6',7'-dihydro-1'H-spiro[indoline-3,2'-pyrazolo[1,2-a]pyrazole]-2,5'(3'H)dione $(4 \mathrm{~g})$

Yield: $118 \mathrm{mg}$ (78\%); yellow solid; mp $166-168{ }^{\circ} \mathrm{C}$.

${ }^{1} \mathrm{H}$ NMR $\left(400 \mathrm{MHz}, \mathrm{CDCl}_{3}\right): \delta=7.15(\mathrm{t}, J=7.2 \mathrm{~Hz}, 1 \mathrm{H}), 7.09-7.04(\mathrm{~m}, 3$ $\mathrm{H}), 6.99(\mathrm{q}, J=8.0 \mathrm{~Hz}, 4 \mathrm{H}), 6.84(\mathrm{t}, J=7.6 \mathrm{~Hz}, 1 \mathrm{H}), 6.51(\mathrm{~d}, J=7.2 \mathrm{~Hz}, 1$ H), $6.46(\mathrm{~d}, J=7.2 \mathrm{~Hz}, 2 \mathrm{H}), 6.34(\mathrm{~d}, J=8.0 \mathrm{~Hz}, 1 \mathrm{H}), 6.02(\mathrm{~s}, 1 \mathrm{H}), 5.12$ $(\mathrm{d}, J=16.0 \mathrm{~Hz}, 1 \mathrm{H}), 4.30(\mathrm{~d}, J=16.0 \mathrm{~Hz}, 1 \mathrm{H}), 4.19(\mathrm{~s}, 1 \mathrm{H}), 3.71(\mathrm{t}, J=$ 8.4 Hz, $1 \mathrm{H}), 3.34-3.19$ ( $\mathrm{m}, 1 \mathrm{H}), 3.05-2.93(\mathrm{~m}, 1 \mathrm{H}), 2.86(\mathrm{~m}, 1 \mathrm{H}), 2.48$ (s, $3 \mathrm{H}), 2.34$ (s, $3 \mathrm{H})$.

${ }^{13} \mathrm{C}$ NMR $\left(125 \mathrm{MHz}, \mathrm{CDCl}_{3}\right): \delta=173.85,167.75,164.28,156.08$, $143.54,139.02,134.63,131.02,130.25,129.39,128.47,127.62$, 127.47, 127.23, 126.42, 123.07, 122.91, 122.41, 109.99, 76.98, 67.49, $54.59,52.25,43.94,36.65,21.33,11.29$.

1-Allyl-3'-(3-methyl-4-nitroisoxazol-5-yl)-1'-(p-tolyl)-6',7'-dihydro-1'H-spiro[indoline-3,2'-pyrazolo[1,2-a]pyrazole]-2,5'(3'H)-dione (4h)

Yield: $128 \mathrm{mg}$ (80\%); white solid; $\mathrm{mp} 151-153^{\circ} \mathrm{C}$.

${ }^{1} \mathrm{H}$ NMR $\left(400 \mathrm{MHz}, \mathrm{CDCl}_{3}\right): \delta=7.20(\mathrm{t}, J=7.6 \mathrm{~Hz}, 1 \mathrm{H}), 6.98(\mathrm{~d}, J=8.0$ $\mathrm{Hz}, 2 \mathrm{H}), 6.95-6.85(\mathrm{~m}, 3 \mathrm{H}), 6.57(\mathrm{~d}, J=8.0 \mathrm{~Hz}, 1 \mathrm{H}), 6.49(\mathrm{~d}, J=7.6 \mathrm{~Hz}$, $1 \mathrm{H}), 6.00(\mathrm{~s}, 1 \mathrm{H}), 5.33-5.22(\mathrm{~m}, 1 \mathrm{H}), 4.85(\mathrm{~d}, J=10.4 \mathrm{~Hz}, 1 \mathrm{H}), 4.42-$ $4.27(\mathrm{~m}, 2 \mathrm{H}), 4.15(\mathrm{~s}, 1 \mathrm{H}), 3.85(\mathrm{dd}, J=16.4,5.2 \mathrm{~Hz}, 1 \mathrm{H}), 3.71(\mathrm{t}, J=$ $8.8 \mathrm{~Hz}, 1 \mathrm{H}), 3.32-3.19(\mathrm{~m}, 1 \mathrm{H}), 3.04-2.93(\mathrm{~m}, 1 \mathrm{H}), 2.86$ (dd, $J=15.8$, $8.0 \mathrm{~Hz}, 1 \mathrm{H}), 2.51(\mathrm{~s}, 3 \mathrm{H}), 2.27(\mathrm{~s}, 3 \mathrm{H})$.

${ }^{13} \mathrm{C}$ NMR $\left(100 \mathrm{MHz}, \mathrm{CDCl}_{3}\right): \delta=173.36,167.82,164.23,156.09$, $143.56,139.07,130.99,130.30,130.16,129.12,127.39,127.28$, 123.04, 122.88, 122.36, 116.63, 109.66, 77.27, 67.53, 54.13, 52.20, 42.19, 36.64, 21.03, 11.30 .

MS (ESI): $m / z$ calcd for $\mathrm{C}_{27} \mathrm{H}_{25} \mathrm{~N}_{5} \mathrm{O}_{5}$ : 499.1856; found: 500.1868 [M + 1].

1-Allyl-1'-(4-methoxyphenyl)-3'-(3-methyl-4-nitroisoxazol-5-yl)6',7'-dihydro-1'H-spiro[indoline-3,2'-pyrazolo[1,2-a]pyrazole]2,5'(3'H)-dione (4i)

Yield: $130 \mathrm{mg}$ (79\%); white solid; $\mathrm{mp} 145-147^{\circ} \mathrm{C}$.

${ }^{1} \mathrm{H} \mathrm{NMR}\left(400 \mathrm{MHz}, \mathrm{CDCl}_{3}\right): \delta=7.20(\mathrm{t}, J=7.6 \mathrm{~Hz}, 1 \mathrm{H}), 6.98(\mathrm{~d}, J=8.8$ $\mathrm{Hz}, 2 \mathrm{H}), 6.89(\mathrm{t}, J=7.6 \mathrm{~Hz}, 1 \mathrm{H}), 6.70(\mathrm{~d}, J=8.8 \mathrm{~Hz}, 2 \mathrm{H}), 6.59(\mathrm{~d}, J=8.0$ $\mathrm{Hz}, 1 \mathrm{H}), 6.48(\mathrm{~d}, J=7.6 \mathrm{~Hz}, 1 \mathrm{H}), 6.01(\mathrm{~s}, 1 \mathrm{H}), 5.41-5.27(\mathrm{~m}, 1 \mathrm{H}), 4.91$ $(\mathrm{d}, J=10.4 \mathrm{~Hz}, 1 \mathrm{H}), 4.50(\mathrm{~d}, J=17.2 \mathrm{~Hz}, 1 \mathrm{H}), 4.34-4.25(\mathrm{~m}, 1 \mathrm{H}), 4.19$ (s, $1 \mathrm{H}), 3.97-3.85(\mathrm{~m}, 1 \mathrm{H}), 3.74(\mathrm{~s}, 3 \mathrm{H}), 3.70(\mathrm{~d}, J=8.4 \mathrm{~Hz}, 1 \mathrm{H}), 3.30-$ $3.18(\mathrm{~m}, 1 \mathrm{H}), 3.07-2.95(\mathrm{~m}, 1 \mathrm{H}), 2.88(\mathrm{dd}, J=16.0,7.6 \mathrm{~Hz}, 1 \mathrm{H}), 2.51$ (s, $3 \mathrm{H})$. 
${ }^{13} \mathrm{C}$ NMR $\left(100 \mathrm{MHz}, \mathrm{CDCl}_{3}\right): \delta=173.42,167.82,164.23,160.32$, $156.10,143.54,130.98,130.32,130.16,128.73,123.01,122.91$, $122.37,122.11,116.81,113.88,109.68,77.23,67.49,55.25,54.13$, 52.19, 42.22, 36.62, 11.31 .

MS (ESI): $m / z$ calcd for $\mathrm{C}_{27} \mathrm{H}_{25} \mathrm{~N}_{5} \mathrm{O}_{6}: 515.1805$; found: 516.1817 [M + 1].

5-Chloro-1-ethyl-3'-(3-methyl-4-nitroisoxazol-5-yl)-1'-phenyl6',7'-dihydro-1'H-spiro[indoline-3,2'-pyrazolo[1,2-a]pyrazole]2,5'(3'H)-dione (4j)

Yield: $123 \mathrm{mg}$ (81\%); light-yellow solid; $\mathrm{mp} 164-166{ }^{\circ} \mathrm{C}$.

${ }^{1} \mathrm{H}$ NMR (400 MHz, $\left.\mathrm{CDCl}_{3}\right): \delta=7.25(\mathrm{~d}, J=7.2 \mathrm{~Hz}, 1 \mathrm{H}), 7.24-7.16(\mathrm{~m}, 3$ H), $7.05(\mathrm{~d}, J=7.2 \mathrm{~Hz}, 2 \mathrm{H}), 6.53(\mathrm{~d}, J=8.4 \mathrm{~Hz}, 1 \mathrm{H}), 6.41(\mathrm{~s}, 1 \mathrm{H}), 5.96$ $(\mathrm{s}, 1 \mathrm{H}), 4.10(\mathrm{~s}, 1 \mathrm{H}), 3.72(\mathrm{t}, J=8.4 \mathrm{~Hz}, 1 \mathrm{H}), 3.65-3.54(\mathrm{~m}, 1 \mathrm{H}), 3.30-$ 3.21 (m, 2 H), 3.04-2.94 (m, 1 H), 2.92-2.83 (m, 1 H), 2.55 (s, $3 \mathrm{H}$ ), $0.66(\mathrm{t}, J=7.2 \mathrm{~Hz}, 3 \mathrm{H})$.

${ }^{13} \mathrm{C}$ NMR $\left(100 \mathrm{MHz}, \mathrm{CDCl}_{3}\right): \delta=172.63,167.52,164.25,156.23$, $141.98,131.07,130.22,130.03,129.26,128.44,127.51,127.37$, 124.82, 123.67, 109.59, 77.19, 67.55, 53.89, 52.16, 36.63, 34.71, 11.53, 11.15.

MS (ESI): $m / z$ calcd for $\mathrm{C}_{25} \mathrm{H}_{22} \mathrm{ClN}_{5} \mathrm{O}_{5}$ : 507.1309; found: 508.1324 [M + 1].

1-Benzyl-5-fluoro-3'-(3-methyl-4-nitroisoxazol-5-yl)-1'-phenyl6',7'-dihydro-1'H-spiro[indoline-3,2'-pyrazolo[1,2-a]pyrazole]2,5'(3'H)-dione (4k)

Yield: $110 \mathrm{mg}$ (76\%); white solid; $\mathrm{mp} 159-161{ }^{\circ} \mathrm{C}$.

${ }^{1} \mathrm{H} \mathrm{NMR}\left(400 \mathrm{MHz}, \mathrm{CDCl}_{3}\right): \delta=7.32-7.27(\mathrm{~m}, 1 \mathrm{H}), 7.16(\mathrm{t}, J=7.6 \mathrm{~Hz}, 2$ H), 7.08-6.99 (m, $5 \mathrm{H}), 6.74-6.68(\mathrm{~m}, 1 \mathrm{H}), 6.33$ (d, J = 7.2 Hz, $2 \mathrm{H})$, 6.25-6.17 (m, $2 \mathrm{H}), 5.96(\mathrm{~s}, 1 \mathrm{H}), 4.98(\mathrm{~d}, J=16.0 \mathrm{~Hz}, 1 \mathrm{H}), 4.23(\mathrm{~d}, J=$ $16.0 \mathrm{~Hz}, 1 \mathrm{H}), 4.11(\mathrm{~s}, 1 \mathrm{H}), 3.66(\mathrm{t}, J=8.4 \mathrm{~Hz}, 1 \mathrm{H}), 3.25-3.13(\mathrm{~m}, 1 \mathrm{H})$, 2.98-2.87 (m, $1 \mathrm{H}), 2.84-2.74(\mathrm{~m}, 1 \mathrm{H}), 2.45(\mathrm{~s}, 3 \mathrm{H})$.

${ }^{13} \mathrm{C}$ NMR $\left(100 \mathrm{MHz}, \mathrm{CDCl}_{3}\right): \delta=173.47,167.32,164.32,159.76$, $157.34,156.19,139.53,134.18,130.27,129.37,128.83,128.72$, 127.72, 127.40, 126.27, 116.94, 116.71, 111.27, 111.02, 77.16, 67.72, $54.44,52.24,44.05,36.61,11.25$.

MS (ESI): $m / z$ calcd for $\mathrm{C}_{30} \mathrm{H}_{24} \mathrm{FN}_{5} \mathrm{O}_{5}: 553.1761$; found: 554.1669 [M + 1].

6-(3-Methyl-4-nitroisoxazol-5-yl)-5,7-diphenyltetrahydropyrazolo[1,2-a]pyrazol-1(5H)-one (6a)

Yield: $131 \mathrm{mg}$ (75\%); white solid; mp $142-144{ }^{\circ} \mathrm{C}$.

${ }^{1} \mathrm{H}$ NMR $\left(400 \mathrm{MHz}, \mathrm{CDCl}_{3}\right): \delta=7.36(\mathrm{~m}, 4 \mathrm{H}), 7.32-7.26(\mathrm{~m}, 1 \mathrm{H}), 7.14$ $(\mathrm{m}, 3 \mathrm{H}), 7.07-7.02(\mathrm{~m}, 2 \mathrm{H}), 5.53(\mathrm{~d}, J=4.0 \mathrm{~Hz}, 1 \mathrm{H}), 4.88(\mathrm{dd}, J=6.8$, $4.8 \mathrm{~Hz}, 1 \mathrm{H}), 4.51(\mathrm{~d}, J=6.8 \mathrm{~Hz}, 1 \mathrm{H}), 3.32(\mathrm{t}, J=10.4 \mathrm{~Hz}, 1 \mathrm{H}), 3.05(\mathrm{dd}$, $J=20.0,10.0 \mathrm{~Hz}, 1 \mathrm{H}), 2.72-2.58(\mathrm{~m}, 2 \mathrm{H}), 2.28(\mathrm{~s}, 3 \mathrm{H})$.

${ }^{13} \mathrm{C}$ NMR $\left(125 \mathrm{MHz}, \mathrm{CDCl}_{3}\right): \delta=167.32,155.89,148.02,144.92$, $132.07,130.65,129.36,128.98,128.07,127.80,127.52,124.53,70.45$, $57.03,56.75,49.65,35.12,10.59$.

MS (ESI): $m / z$ calcd for $\mathrm{C}_{22} \mathrm{H}_{20} \mathrm{~N}_{4} \mathrm{O}_{4}$ : 404.1485; found: 405.1558 [M + 1].

\section{5-(Furan-2-yl)-6-(3-methyl-4-nitroisoxazol-5-yl)-7-phenyltetra-} hydropyrazolo[1,2-a]pyrazol-1(5H)-one (6b)

Yield: $154 \mathrm{mg}$ (90\%); yellow semi-solid.

${ }^{1} \mathrm{H}$ NMR (400 MHz, $\mathrm{CDCl}_{3}$ ): $\delta=7.42(\mathrm{~d}, J=7.6 \mathrm{~Hz}, 2 \mathrm{H}), 7.33(\mathrm{~s}, 1 \mathrm{H})$, 7.29 (t, $J=7.6 \mathrm{~Hz}, 2 \mathrm{H}), 7.23(\mathrm{~d}, J=7.2 \mathrm{~Hz}, 1 \mathrm{H}), 6.25-6.20(\mathrm{~m}, 2 \mathrm{H})$, $6.02(\mathrm{~d}, J=8.4 \mathrm{~Hz}, 1 \mathrm{H}), 4.99(\mathrm{~d}, J=6.8 \mathrm{~Hz}, 1 \mathrm{H}), 4.79-4.74(\mathrm{~m}, 1 \mathrm{H})$, $3.55(\mathrm{dd}, J=22.0,10.0 \mathrm{~Hz}, 1 \mathrm{H}), 3.12(\mathrm{~m}, 1 \mathrm{H}), 2.39(\mathrm{~s}, 3 \mathrm{H}), 1.78-1.61$ ( $\mathrm{m}, 2 \mathrm{H})$.

MS (ESI): $m / z$ calcd for $\mathrm{C}_{20} \mathrm{H}_{18} \mathrm{~N}_{4} \mathrm{O}_{5}$ : 394.1277; found: 395.1369 [M + 1].
6-(3-Methyl-4-nitroisoxazol-5-yl)-7-(4-nitrophenyl)-5-phenyltetrahydropyrazolo[1,2-a]pyrazol-1(5H)-one (6c)

Yield: $133 \mathrm{mg}$ (82\%); white solid; $\mathrm{mp} 182-184{ }^{\circ} \mathrm{C}$.

${ }^{1} \mathrm{H} \mathrm{NMR}\left(400 \mathrm{MHz}, \mathrm{CDCl}_{3}\right): \delta=8.29(\mathrm{~d}, J=8.8 \mathrm{~Hz}, 2 \mathrm{H}), 7.66(\mathrm{~d}, J=8.8$ $\mathrm{Hz}, 2 \mathrm{H}), 7.28(\mathrm{~m}, 3 \mathrm{H}), 7.12(\mathrm{~d}, J=7.6 \mathrm{~Hz}, 2 \mathrm{H}), 5.76(\mathrm{~d}, J=4.0 \mathrm{~Hz}, 1 \mathrm{H})$, 4.97-4.90 (m, $1 \mathrm{H}), 4.66(\mathrm{~d}, J=6.8 \mathrm{~Hz}, 1 \mathrm{H}), 3.41(\mathrm{t}, J=11.2 \mathrm{~Hz}, 1 \mathrm{H})$, 3.22 (dd, $J=20.0,10.0 \mathrm{~Hz}, 1 \mathrm{H}), 2.76-2.63(\mathrm{~m}, 2 \mathrm{H}), 2.38$ (s, $3 \mathrm{H})$.

${ }^{13} \mathrm{C}$ NMR $\left(125 \mathrm{MHz}, \mathrm{CDCl}_{3}\right): \delta=168.90,155.89,148.02,144.92$, 132.07, 130.65, 129.36, 128.98, 128.07, 127.80, 127.52, 124.53, 70.45, 57.03, 56.75, 47.61, 35.12, 11.37 .

MS (ESI): $m / z$ calcd for $\mathrm{C}_{22} \mathrm{H}_{19} \mathrm{~N}_{5} \mathrm{O}_{6}: 449.1335$; found: 450.1403 [M + 1].

6-(3-Methyl-4-nitroisoxazol-5-yl)-7-(4-nitrophenyl)-5(p-tolyl)tetrahydropyrazolo[1,2-a]pyrazol-1(5H)-one (6d) Yield: $117 \mathrm{mg}$ (70\%); white solid; mp $179-181{ }^{\circ} \mathrm{C}$.

${ }^{1} \mathrm{H}$ NMR $\left(400 \mathrm{MHz}, \mathrm{CDCl}_{3}\right): \delta=8.27(\mathrm{~d}, J=8.8 \mathrm{~Hz}, 2 \mathrm{H}), 7.64(\mathrm{~d}, J=8.8$ $\mathrm{Hz}, 2 \mathrm{H}), 7.05(\mathrm{~d}, J=8.0 \mathrm{~Hz}, 2 \mathrm{H}), 6.97(\mathrm{~d}, J=8.0 \mathrm{~Hz}, 2 \mathrm{H}), 5.79(\mathrm{~d}, J=4.4$ $\mathrm{Hz}, 1 \mathrm{H}), 4.91-4.84(\mathrm{~m}, 1 \mathrm{H}), 4.66(\mathrm{~d}, J=6.8 \mathrm{~Hz}, 1 \mathrm{H}), 3.33(\mathrm{t}, J=10.4$ $\mathrm{Hz}, 1 \mathrm{H}), 3.23(\mathrm{dd}, J=19.2,9.6 \mathrm{~Hz}, 1 \mathrm{H}), 2.61(\mathrm{~m}, 2 \mathrm{H}), 2.38(\mathrm{~s}, 3 \mathrm{H})$, $2.28(\mathrm{~s}, 3 \mathrm{H})$.

${ }^{13} \mathrm{C}$ NMR $\left(125 \mathrm{MHz}, \mathrm{CDCl}_{3}\right): \delta=168.97,155.97,147.99,139.40$, 130.66, 129.71, 128.97, 128.15, 127.45, 124.52, 124.42, 114.91, 70.15, $57.10,56.64,34.70,29.71,21.12,11.43$.

MS (ESI): $m / z$ calcd for $\mathrm{C}_{23} \mathrm{H}_{21} \mathrm{~N}_{5} \mathrm{O}_{6}: 463.1492$; found: 464.1566 [M + 1].

5-(Furan-2-yl)-6-(3-methyl-4-nitroisoxazol-5-yl)-7-(4-nitrophenyl)tetrahydropyrazolo[1,2-a]pyrazol-1(5H)-one (6e)

Yield: $135 \mathrm{mg}$ (85\%); light-yellow solid; $\mathrm{mp} 175-177^{\circ} \mathrm{C}$.

${ }^{1} \mathrm{H} \mathrm{NMR}\left(400 \mathrm{MHz}, \mathrm{CDCl}_{3}\right): \delta=8.15(\mathrm{~d}, J=8.8 \mathrm{~Hz}, 2 \mathrm{H}), 7.60(\mathrm{~d}, J=8.4$ $\mathrm{Hz}, 2 \mathrm{H}), 7.34(\mathrm{~s}, 1 \mathrm{H}), 6.25(\mathrm{~m}, 2 \mathrm{H}), 6.11(\mathrm{~d}, J=8.4 \mathrm{~Hz}, 1 \mathrm{H}), 5.01(\mathrm{~d}, J=$ $6.8 \mathrm{~Hz}, 1 \mathrm{H}), 4.71-4.65(\mathrm{~m}, 1 \mathrm{H}), 3.59(\mathrm{dd}, J=22.0,9.6 \mathrm{~Hz}, 1 \mathrm{H}), 3.20-$ $3.10(\mathrm{~m}, 1 \mathrm{H}), 2.41(\mathrm{~s}, 3 \mathrm{H}), 2.38-2.32(\mathrm{~m}, 1 \mathrm{H}), 1.64(\mathrm{~m}, 1 \mathrm{H})$.

${ }^{13} \mathrm{C}$ NMR $\left(125 \mathrm{MHz}, \mathrm{CDCl}_{3}\right): \delta=168.97,155.97,147.99,139.40$, 130.66, 129.71, 128.97, 128.15, 127.45, 124.52, 124.42, 114.91, 70.15, $57.10,56.64,29.71,21.12,11.43$.

MS (ESI): $m / z$ calcd for $\mathrm{C}_{20} \mathrm{H}_{17} \mathrm{~N}_{5} \mathrm{O}_{7}: 439.1128$; found: 440.1188 [M + 1].

6-(3-Methyl-4-nitroisoxazol-5-yl)-5-phenyl-7-(p-tolyl)tetrahydropyrazolo[1,2-a]pyrazol-1(5H)-one (6f)

Yield: $119 \mathrm{mg}$ (70\%); white solid; mp $164-166{ }^{\circ} \mathrm{C}$.

${ }^{1} \mathrm{H} \mathrm{NMR}\left(400 \mathrm{MHz}, \mathrm{CDCl}_{3}\right): \delta=7.33(\mathrm{~d}, J=7.6 \mathrm{~Hz}, 2 \mathrm{H}), 7.22(\mathrm{~m}, 5 \mathrm{H})$, 7.14-7.09 (m, $2 \mathrm{H}), 5.53(\mathrm{~d}, J=3.6 \mathrm{~Hz}, 1 \mathrm{H}), 4.94(\mathrm{dd}, J=7.2,4.8 \mathrm{~Hz}, 1$ $\mathrm{H}), 4.54(\mathrm{~d}, J=7.2 \mathrm{~Hz}, 1 \mathrm{H}), 3.39(\mathrm{t}, J=8.4 \mathrm{~Hz}, 1 \mathrm{H}), 3.07$ (dd, $J=20.4$, $9.6 \mathrm{~Hz}, 1 \mathrm{H}), 2.86-2.65$ (m, $2 \mathrm{H}), 2.36$ (s, $3 \mathrm{H}), 2.34$ (s, $3 \mathrm{H})$.

${ }^{13} \mathrm{C}$ NMR $\left(125 \mathrm{MHz}, \mathrm{CDCl}_{3}\right): \delta=169.00,154.53,137.51,133.66$, 131.54, 129.50, 129.01, 128.89, 127.98, 127.68, 126.84, 125.32, 69.35, $56.76,56.25,34.97,28.68,20.15,10.36$.

MS (ESI): $m / z$ calcd for $\mathrm{C}_{23} \mathrm{H}_{22} \mathrm{~N}_{4} \mathrm{O}_{4}: 418.1641$; found: 419.1713 [M + 1].

5-(Furan-2-yl)-6-(3-methyl-4-nitroisoxazol-5-yl)-7-(p-tolyl)tetrahydropyrazolo[1,2-a]pyrazol-1(5H)-one $(6 \mathrm{~g})$

Yield: $122 \mathrm{mg}$ (73\%); light-yellow semi-solid.

${ }^{1} \mathrm{H}$ NMR $\left(400 \mathrm{MHz}, \mathrm{CDCl}_{3}\right): \delta=7.39(\mathrm{~d}, J=3.2 \mathrm{~Hz}, 2 \mathrm{H}), 7.36(\mathrm{~s}, 1 \mathrm{H})$, $7.16(\mathrm{~d}, J=8.0 \mathrm{~Hz}, 2 \mathrm{H}), 6.31-6.28(\mathrm{~m}, 1 \mathrm{H}), 6.26(\mathrm{~d}, J=3.2 \mathrm{~Hz}, 1 \mathrm{H})$, $6.04(\mathrm{~d}, J=8.4 \mathrm{~Hz}, 1 \mathrm{H}), 5.03(\mathrm{~d}, J=6.8 \mathrm{~Hz}, 1 \mathrm{H}), 4.82(\mathrm{dd}, J=8.0,6.8 \mathrm{~Hz}$, $1 \mathrm{H}), 3.59$ (dd, $J=22.0,10.0 \mathrm{~Hz}, 1 \mathrm{H}), 3.21-3.13(\mathrm{~m}, 1 \mathrm{H}), 2.47(\mathrm{~s}, 1 \mathrm{H})$, 2.45 (s, $3 \mathrm{H}), 2.44-2.38(\mathrm{~m}, 1 \mathrm{H}), 2.32(\mathrm{~s}, 3 \mathrm{H})$. 
${ }^{13} \mathrm{C}$ NMR $\left(125 \mathrm{MHz}, \mathrm{CDCl}_{3}\right): \delta=174.34,168.90,156.06,147.18$, $144.10,138.08,136.60,130.71,129.73,126.15,112.20,111.19,62.26$, $57.54,55.05,31.45,29.71,21.13,11.52$.

MS (ESI): $m / z$ calcd for $\mathrm{C}_{21} \mathrm{H}_{20} \mathrm{~N}_{4} \mathrm{O}_{5}$ : 408.1434; found: 409.1505 [M + 1].

7-(4-Chlorophenyl)-6-(3-methyl-4-nitroisoxazol-5-yl)-5-phenyltetrahydropyrazolo[1,2-a]pyrazol-1(5H)-one (6h)

Yield: $126 \mathrm{mg}$ (76\%); white solid; $\mathrm{mp} 148-150{ }^{\circ} \mathrm{C}$.

${ }^{1} \mathrm{H}$ NMR (400 MHz, $\mathrm{CDCl}_{3}$ ): $\delta=7.39(\mathrm{~s}, 4 \mathrm{H}), 7.22(\mathrm{~m}, 3 \mathrm{H}), 7.13-7.08$ $(\mathrm{m}, 2 \mathrm{H}), 5.57(\mathrm{~d}, J=4.0 \mathrm{~Hz}, 1 \mathrm{H}), 4.91(\mathrm{dd}, J=7.2,4.8 \mathrm{~Hz}, 1 \mathrm{H}), 4.56$ (d, $J=7.2 \mathrm{~Hz}, 1 \mathrm{H}), 3.39$ (m, $1 \mathrm{H}), 3.11$ (q, J = $10.0 \mathrm{~Hz}, 1 \mathrm{H}), 2.70$ (m, $2 \mathrm{H})$, $2.35(\mathrm{~s}, 3 \mathrm{H})$

${ }^{13} \mathrm{C}$ NMR $\left(125 \mathrm{MHz}, \mathrm{CDCl}_{3}\right): \delta=169.50,155.73,136.21,134.65$, $132.13,130.60,129.48,129.27,128.90,128.01,127.90,70.35,57.37$, $56.97,35.40,29.71,11.38$.

MS (ESI): $m / z$ calcd for $\mathrm{C}_{22} \mathrm{H}_{19} \mathrm{ClN}_{4} \mathrm{O}_{4}: 438.1095$; found: 439.1161 [M + 1].

6-(3-Methyl-4-nitroisoxazol-5-yl)-7-(naphthalen-1-yl)-5-phenyltetrahydropyrazolo[1,2-a]pyrazol-1(5H)-one (6i)

Yield: $113 \mathrm{mg}$ (70\%); white solid; $\mathrm{mp} 151-153^{\circ} \mathrm{C}$.

${ }^{1} \mathrm{H}$ NMR $\left(400 \mathrm{MHz}, \mathrm{CDCl}_{3}\right): \delta=7.90(\mathrm{t}, J=8.8 \mathrm{~Hz}, 2 \mathrm{H}), 7.75(\mathrm{~d}, J=7.2$ $\mathrm{Hz}, 1 \mathrm{H}), 7.63(\mathrm{~d}, J=8.4 \mathrm{~Hz}, 1 \mathrm{H}), 7.57(\mathrm{t}, J=7.6 \mathrm{~Hz}, 1 \mathrm{H}), 7.49(\mathrm{t}, J=6.8$ $\mathrm{Hz}, 1 \mathrm{H}), 7.43(\mathrm{t}, J=7.2 \mathrm{~Hz}, 1 \mathrm{H}), 7.24-7.17(\mathrm{~m}, 3 \mathrm{H}), 7.09(\mathrm{~d}, J=6.8 \mathrm{~Hz}$, $2 \mathrm{H}), 6.24(\mathrm{~d}, J=2.8 \mathrm{~Hz}, 1 \mathrm{H}), 4.96(\mathrm{dd}, J=6.4,3.6 \mathrm{~Hz}, 1 \mathrm{H}), 4.47(\mathrm{~d}, J=$ $6.8 \mathrm{~Hz}, 1 \mathrm{H}), 3.52(\mathrm{~m}, 1 \mathrm{H}), 3.11$ (m, $2 \mathrm{H}), 2.86(\mathrm{~m}, 1 \mathrm{H}), 2.40$ (s, $3 \mathrm{H})$.

${ }^{13} \mathrm{C}$ NMR $\left(125 \mathrm{MHz}, \mathrm{CDCl}_{3}\right): \delta=170.05,165.10,155.60,134.33$, $132.21,132.17,130.53,129.99,129.44,129.32,129.05,128.62$, 127.59, 127.06, 126.06, 125.32, 123.92, 121.71, 69.74, 56.61, 55.18, $36.85,29.72,11.47$

MS (ESI): $m / z$ calcd for $\mathrm{C}_{26} \mathrm{H}_{22} \mathrm{~N}_{4} \mathrm{O}_{4}: 454.1641$; found: 455.1701 [M + 1].

6-(3-Methyl-4-nitroisoxazol-5-yl)-7-(4-oxo-4H-chromen-3-yl)-5phenyltetrahydropyrazolo[1,2-a]pyrazol-1(5H)-one (6j)

Yield: $123 \mathrm{mg}$ (78\%); white solid; $\mathrm{mp} 180-182{ }^{\circ} \mathrm{C}$.

${ }^{1} \mathrm{H}$ NMR $\left(400 \mathrm{MHz}, \mathrm{CDCl}_{3}\right): \delta=8.12(\mathrm{dd}, J=8.0,1.2 \mathrm{~Hz}, 1 \mathrm{H}), 8.02(\mathrm{~s}, 1$ $\mathrm{H}), 7.68-7.62(\mathrm{~m}, 1 \mathrm{H}), 7.44(\mathrm{~d}, J=8.4 \mathrm{~Hz}, 1 \mathrm{H}), 7.37(\mathrm{t}, J=7.6 \mathrm{~Hz}, 1 \mathrm{H})$, $7.12(\mathrm{~m}, 5 \mathrm{H}), 5.24(\mathrm{~d}, J=3.2 \mathrm{~Hz}, 1 \mathrm{H}), 5.14(\mathrm{dd}, J=7.2,3.6 \mathrm{~Hz}, 1 \mathrm{H})$, 4.63 (d, J = 7.2 Hz, $1 \mathrm{H}$ ), 3.43 (m, $1 \mathrm{H}), 2.98-2.86$ (m, $2 \mathrm{H}), 2.73$ (m, 1 $\mathrm{H}), 2.27(\mathrm{~s}, 3 \mathrm{H})$

${ }^{13} \mathrm{C}$ NMR $\left(125 \mathrm{MHz}, \mathrm{CDCl}_{3}\right): \delta=175.42,169.82,164.87,155.34$, $154.37,153.22,133.22,131.71,129.30,127.78,127.48,126.45$, $124.65,122.75,118.23,117.24,69.84,52.71,52.31,48.24,35.34$, 10.37 .

MS (ESI): $m / z$ calcd for $\mathrm{C}_{25} \mathrm{H}_{20} \mathrm{~N}_{4} \mathrm{O}_{6}$ : 472.1383; found: 473.1453 [M + 1].

\section{2-(2-(3-Methyl-4-nitroisoxazol-5-yl)-7-oxo-3-phenylhexahydro-} pyrazolo[1,2-a]pyrazol-1-yl)phenyl 4-nitrobenzenesulfonate (6k) Yield: $170 \mathrm{mg}$ (65\%); light-yellow solid; mp $159-161{ }^{\circ} \mathrm{C}$

${ }^{1} \mathrm{H}$ NMR $\left(400 \mathrm{MHz}, \mathrm{CDCl}_{3}\right): \delta=8.41(\mathrm{~d}, J=8.8 \mathrm{~Hz}, 2 \mathrm{H}), 8.16(\mathrm{~d}, J=8.8$ $\mathrm{Hz}, 2 \mathrm{H}), 7.46(\mathrm{~d}, J=4.0 \mathrm{~Hz}, 5 \mathrm{H}), 7.32(\mathrm{dd}, J=5.4,1.9 \mathrm{~Hz}, 1 \mathrm{H}), 7.29$ (d, $J=1.6 \mathrm{~Hz}, 1 \mathrm{H}), 7.24(\mathrm{dd}, J=11.0,4.0 \mathrm{~Hz}, 1 \mathrm{H}), 7.06(\mathrm{~d}, J=8.0 \mathrm{~Hz}, 1 \mathrm{H})$, $5.48(\mathrm{~d}, J=2.4 \mathrm{~Hz}, 1 \mathrm{H}), 5.01(\mathrm{~d}, J=6.4 \mathrm{~Hz}, 1 \mathrm{H}), 4.99-4.95(\mathrm{~m}, 1 \mathrm{H})$, $3.51(\mathrm{t}, J=8.6 \mathrm{~Hz}, 1 \mathrm{H}), 3.21$ (dd, $J=20.2,9.8 \mathrm{~Hz}, 1 \mathrm{H}), 3.05-2.99(\mathrm{~m}, 1$ $\mathrm{H}), 2.82(\mathrm{dd}, J=15.2,8.0 \mathrm{~Hz}, 1 \mathrm{H}), 2.39(\mathrm{~s}, 3 \mathrm{H})$.
${ }^{13} \mathrm{C}$ NMR $\left(100 \mathrm{MHz}, \mathrm{CDCl}_{3}\right): \delta=169.29,164.87,155.50,151.21$, $147.30,141.14,137.22,130.64,130.36,129.57,129.35,128.89$, $128.59,127.73,126.46,126.43,124.71,122.36,63.57,58.53,55.60$, 49.52, 36.41, 11.37 .

MS (ESI): $m / z$ calcd for $\mathrm{C}_{28} \mathrm{H}_{23} \mathrm{~N}_{5} \mathrm{O}_{9} \mathrm{~S}$ : 605.1216; found: 606.1297 [M + 1].

1-Methyl-2,7'-dioxo-3'-phenyl-3',5',6',7'-tetrahydro-2'H-spiroindoline-3,1'-pyrazolo[1,2-a]pyrazole]-2'-carboxylic Acid (7a) Yield: $64 \mathrm{mg}$ (78\%); cream solid; $\mathrm{mp} 217-219^{\circ} \mathrm{C}$.

${ }^{1} \mathrm{H}$ NMR $\left(400 \mathrm{MHz}\right.$, DMSO- $\left.d_{6}\right): \delta=7.56(\mathrm{~d}, J=7.2 \mathrm{~Hz}, 2 \mathrm{H}), 7.45(\mathrm{~d}, J=$ $7.2 \mathrm{~Hz}, 1 \mathrm{H}), 7.37$ (t, J = 7.2 Hz, $2 \mathrm{H}), 7.32-7.27(\mathrm{~m}, 2 \mathrm{H}), 7.04-6.97$ (m, $2 \mathrm{H}), 4.27$ (d, $J=11.2 \mathrm{~Hz}, 1 \mathrm{H}), 3.68(\mathrm{~d}, J=10.8 \mathrm{~Hz}, 1 \mathrm{H}), 3.15(\mathrm{~s}, 3 \mathrm{H})$, 3.02-2.94 (m, $1 \mathrm{H}), 2.80-2.71(\mathrm{~m}, 1 \mathrm{H}), 2.42-2.29(\mathrm{~m}, 2 \mathrm{H})$.

MS (ESI): $m / z$ calcd for $\mathrm{C}_{21} \mathrm{H}_{19} \mathrm{~N}_{3} \mathrm{O}_{4}: 377.1376$; found: 378.1460 [M + 1].

1-(Furan-2-yl)-5-oxo-3-phenylhexahydropyrazolo[1,2-a]pyrazole2-carboxylic Acid (8b)

Yield: $67 \mathrm{mg}$ (85\%); cream solid; $\mathrm{mp} 233-235^{\circ} \mathrm{C}$.

${ }^{1} \mathrm{H}$ NMR $\left(400 \mathrm{MHz}\right.$, DMSO- $\left.d_{6}\right): \delta=12.78(\mathrm{~s}, 1 \mathrm{H}), 7.71-7.70(\mathrm{~d}, J=8.0$ $\mathrm{Hz}, 1 \mathrm{H}), 7.43$ (d, $J=7.2 \mathrm{~Hz}, 2 \mathrm{H}), 7.38(\mathrm{t}, J=7.2 \mathrm{~Hz}, 2 \mathrm{H}), 7.30$ (t, $J=6.8$ $\mathrm{Hz}, 1 \mathrm{H}), 6.53-6.52(\mathrm{~m}, 1 \mathrm{H}), 6.46(\mathrm{~d}, J=2.8 \mathrm{~Hz}, 1 \mathrm{H}), 5.40(\mathrm{~d}, J=8.4 \mathrm{~Hz}$, $1 \mathrm{H}), 4.66(\mathrm{~d}, J=7.2 \mathrm{~Hz}, 1 \mathrm{H}), 3.85(\mathrm{t}, J=7.6 \mathrm{~Hz}, 1 \mathrm{H}), 3.43(\mathrm{dd}, J=22.4$, $10.0 \mathrm{~Hz}, 1 \mathrm{H}$ ), 3.13-3.07 (m, $1 \mathrm{H}), 2.27-2.20$ (m, 1 H), 1.26-1.17 (m, 1 $\mathrm{H})$.

${ }^{13} \mathrm{C}$ NMR $\left(100 \mathrm{MHz}\right.$, DMSO- $\left.d_{6}\right): \delta=175.41,170.67,149.21,144.03$, 141.93, 129.03, 127.87, 126.62, 111.95, 111.49, 61.24, 59.15, 58.48, $42.70,30.70$

MS (ESI): $m / z$ calcd for $\mathrm{C}_{17} \mathrm{H}_{16} \mathrm{~N}_{2} \mathrm{O}_{4}: 312.1110$; found: 313.1194 [M + 1].

Methyl 1-(Furan-2-yl)-5-oxo-3-phenylhexahydropyrazolo [1,2-a]pyrazole-2-carboxylate (9b)

Yield: $67 \mathrm{mg}$ (65\%); light-yellow liquid.

${ }^{1} \mathrm{H}$ NMR $\left(400 \mathrm{MHz}\right.$, DMSO- $d_{6}$ ): $\delta=7.69$ (d, $\left.J=1.2 \mathrm{~Hz}, 1 \mathrm{H}\right), 7.44-7.42$ $(\mathrm{m}, 2 \mathrm{H}), 7.38(\mathrm{t}, J=7.2 \mathrm{~Hz}, 2 \mathrm{H}), 7.32-7.28(\mathrm{~m}, 1 \mathrm{H}), 6.53(\mathrm{dd}, J=3.6$, $2.0 \mathrm{~Hz}, 1 \mathrm{H}), 6.46(\mathrm{~d}, J=3.2 \mathrm{~Hz}, 1 \mathrm{H}), 5.43(\mathrm{~d}, J=8.0 \mathrm{~Hz}, 1 \mathrm{H}), 4.70(\mathrm{~d}, J=$ $7.2 \mathrm{~Hz}, 1 \mathrm{H}), 3.99(\mathrm{t}, J=8.0 \mathrm{~Hz}, 1 \mathrm{H}), 3.45(\mathrm{~s}, 3 \mathrm{H}), 3.42-3.39(\mathrm{~m}, 1 \mathrm{H})$, 3.15-3.09 (m, $1 \mathrm{H}), 2.28-2.20(\mathrm{~m}, 1 \mathrm{H}), 1.25-1.17(\mathrm{~m}, 1 \mathrm{H})$.

${ }^{13} \mathrm{C}$ NMR $\left(125 \mathrm{MHz}\right.$, DMSO- $\left.d_{6}\right): \delta=174.94,169.37,148.48,143.70$, $141.11,128.61,127.53,126.22,111.52,111.01,60.66,58.20,57.99$, $52.16,42.30,30.20$.

MS (ESI): $m / z$ calcd for $\mathrm{C}_{18} \mathrm{H}_{18} \mathrm{~N}_{2} \mathrm{O}_{4}: 326.1267$; found: 327.1350 [M + 1].

\section{1-(Furan-2-yl)-5-oxo-3-phenyl- $N$-(p-tolyl)hexahydropyrazolo [1,2-a]pyrazole-2-carboxamide (10b)}

Yield: $109 \mathrm{mg}$ (85\%); white solid; $\mathrm{mp} 222-224^{\circ} \mathrm{C}$.

${ }^{1} \mathrm{H}$ NMR (400 MHz, DMSO- $d_{6}$ ): $\delta=9.88(\mathrm{~s}, 1 \mathrm{H}), 7.70(\mathrm{~d}, J=1.2 \mathrm{~Hz}, 1$ H), $7.38(\mathrm{~d}, J=4.4 \mathrm{~Hz}, 4 \mathrm{H}), 7.32-7.28(\mathrm{~m}, 1 \mathrm{H}), 7.24(\mathrm{~d}, J=8.4 \mathrm{~Hz}, 2 \mathrm{H})$, $7.04(\mathrm{~d}, J=8.4 \mathrm{~Hz}, 2 \mathrm{H}), 6.46-6.45(\mathrm{~m}, 1 \mathrm{H}), 6.41(\mathrm{~d}, J=3.2 \mathrm{~Hz}, 1 \mathrm{H})$, $5.64(\mathrm{~d}, J=8.4 \mathrm{~Hz}, 1 \mathrm{H}), 4.85(\mathrm{~d}, J=6.8 \mathrm{~Hz}, 1 \mathrm{H}), 3.82(\mathrm{t}, J=7.6 \mathrm{~Hz}, 1 \mathrm{H})$, 3.45 (dd, $J=22.0,10.0 \mathrm{~Hz}, 1 \mathrm{H}), 3.15-3.08(\mathrm{~m}, 1 \mathrm{H}), 2.35-2.20(\mathrm{~m}, 1 \mathrm{H})$, $2.20(\mathrm{~s}, 3 \mathrm{H}), 1.45-1.33(\mathrm{~m}, 1 \mathrm{H})$.

${ }^{13} \mathrm{C}$ NMR $\left(125 \mathrm{MHz}\right.$, DMSO- $\left.d_{6}\right): \delta=174.60,165.32,148.27,143.99$, $141.59,135.96,132.59,129.04,128.69,127.38,125.83,119.59$, 111.54, 111.12, 61.63, 60.77, 57.35, 42.53, 30.69, 20.40.

MS (ESI): $m / z$ calcd for $\mathrm{C}_{24} \mathrm{H}_{23} \mathrm{~N}_{3} \mathrm{O}_{3}$ : 401.1739; found: 402.1829 [M + 1]. 


\section{Conflict of Interest}

The authors declare no conflict of interest.

\section{Funding Information}

K.S. thanks DST for an INSPIRE fellowship. S.N. thanks UGC-New Delhi for a Fellowship. D.K. thanks DST (SERB), New Delhi for financial support (SB/FT/CS-136/2012 and SB/EMEQ-103/2014).

\section{Supporting Information}

Supporting information for this article is available online at https://doi.org/10.1055/a-1480-9837.

\section{References}

(1) (a) Babu, S. A.; Padmavathi, R.; Aslam, N. A.; Rajkumar, V. Stud. Nat. Prod. Chem. 2015, 46, 227. (b) Singh, G. S.; Desta, Z. Y. Chem. Rev. 2012, 112, 6104. (c) Cheng, D.; Ishihara, Y.; Tan, B.; Barbas, C. F. III ACS Catal. 2014, 4, 743. (d) Zhou, L.-M.; Qu, R.-Y.; Yang, G.-F. Expert Opin. Drug Discovery 2020, 15, 603.

(2) (a) Zhao, Z.; Dai, X.; Li, C.; Wang, X.; Tian, J.; Feng, Y.; Xie, J.; Ma, C.; Nie, Z.; Fan, P.; Qian, M.; He, X.; Wu, S.; Zhang, Y.; Zheng, X. Eur. J. Med. Chem. 2020, 186, 111893. (b) Mrongovius, R.; Neugebauer, M.; Rucker, G. Eur. J. Med. Chem. 1984, 19, 161. (c) Khan, M. F.; Alam, M. M.; Verma, G.; Akhtar, W.; Akhter, M.; Shaquiquzzaman, M. Eur. J. Med. Chem. 2016, 120, 170.

(3) (a) Yet, L. In Comprehensive Heterocyclic Chemistry III, Vol. 4; Katrizky, A. R.; Ramsden, C. A.; Scriven, E. F. V.; Taylor, R. J. K., Ed.; Elsevier Science: Oxford, 2008, 1. (b) Radl, S. In Comprehensive Heterocyclic Chemistry III, Vol. 12; Katrizky, A. R.; Ramsden, C. A.; Scriven, E. F. V.; Taylor, R. J. K., Ed.; Elsevier Science: Oxford, 2008, 1-75. (c) Liu, S.; Bao, X.; Wang, B. Chem. Commun. 2018, 54, 11515.

(4) (a) Pezdirc, L.; Jovanovski, V.; Bevk, D.; Jakše, R.; Pirc, S.; Meden, A.; Stanovnik, B.; Svete, J. Tetrahedron 2005, 61, 3977. (b) Boyd, D. B. J. Med. Chem. 1993, 36, 1443. (c) Kamata, M.; Yamashita, T.; Kina, A.; Tawada, M.; Endo, S.; Mizukami, A.; Sasaki, M.; Tani, A.; Nakano, Y.; Watanabe, Y.; Furuyama, N.; Funami, M.; Amano, N.; Fukatsu, K. Bioorg. Med. Chem. Lett. 2012, 22, 4769. (d) Yao, S.; Gallenkamp, D.; Wölfel, K.; Lüke, B.; Schindler, M.; Scherkenbeck, J. Bioorg. Med. Chem. 2011, 19, 4669. (e) Clark, M. P.; Laughlin, S. K.; Laufersweiler, M. J.; Bookland, R. G.; Brugel, T. A.; Golebiowski, A.; Sabat, M. P.; Townes, J. A.; VanRens, J. C.; Djung, J. F.; Natchus, M. G.; De, B.; Hsieh, L. C.; Xu, S. C.; Walter, R. L.; Mekel, M. J.; Heitmeyer, S. A.; Brown, K. K.; Juergens, K.; Taiwo, Y. O.; Janusz, M. J. J. Med. Chem. 2004, 47, 2724.
(5) (a) Muehlebach, M.; Boeger, M.; Cederbaum, F.; Cornes, D.; Friedmann, A. A.; Glock, J.; Niderman, T.; Stoller, A.; Wagner, T. Bioorg. Med. Chem. 2009, 17, 4241. (b) Varvounis, G.; Fiamegos, Y.; Pilidis, G. Adv. Heterocycl. Chem. 2001, 80, 75.

(6) (a) Funken, N.; Zhang, Y.-Q.; Gans?uer, A. Chem. Eur. J. 2017, 23, 19. (b) Engler, T. A.; Chai, W.; Lynch, K. O. Jr. Tetrahedron Lett. 1995, 36, 7003. (c) Zhan, G.; Du, W.; Chen, Y.-C. Chem. Soc. Rev. 2017, 46, 1675. (d) Zhou, Z.; Wang, Z.-X.; Zhou, Y.-C.; Xiao, W.; Ouyang, Q.; Du, W.; Chen, Y.-C. Nat. Chem. 2017, 9, 590. (e) Nagaraju, S.; Liu, S.; Liu, J.; Yang, S.; Liu, R.; Chen, Z.; Paplal, B.; Fang, X. Org. Lett. 2019, 21, 10075. (f) Meng, J. P.; Wang, W. W.; Chen, Y. L.; Bera, S.; Wu, J. Org. Chem. Front. 2020, 7, 267. (g) Chavan, S. P.; Varadwaj, G. B.; Parida, K. M.; Bhanage, B. M. ChemCatChem 2016, 8, 2649.

(7) (a) Merino, P.; Tejero, T.; Díez-Martínez, A.; Gültekin, Z. Eur. J. Org. Chem. 2011, 6567. (b) Chen, Y. R.; Zhan, G.; Du, W.; Chen, Y.-C. Adv. Synth. Catal. 2016, 358, 3759. (c) Tong, M.; Zhang, Y.; Qin, C.; Fu, Y.; Liu, Y.; Li, H.; Wang, W. Org. Chem. Front. 2018, 5, 2945. (d) Qiu, G.; Kuang, Y.; Wu, J. Adv. Synth. Catal. 2014, 356, 3483. (e) Nájera, C.; Sansano, J. M.; Yus, M. Org. Biomol. Chem. 2015, 13, 8596.

(8) (a) Narayan, R.; Potowski, M.; Jia, Z.-J.; Antonchick, A. P.; Waldmann, H. Acc. Chem. Res. 2014, 47, 1296. (b) Adrio, J.; Carretero, J. C. Chem. Commun. 2014, 50, 12434. (c) Bdiri, B.; Zhao, B.-J.; Zhou, Z.-M. Tetrahedron: Asymmetry 2017, 28, 876. (d) Vishwanath, M.; Sivamuthuraman, K.; Kesavan, V. Chem. Commun. 2016, 52, 12314.

(9) (a) Hong, L.; Kai, M.; Wu, C.; Sun, W.; Zhu, G.; Li, G.; Yao, X.; Wang, R. Chem. Commun. 2013, 49, 6713. (b) Yin, C.; Lin, L.; Zhang, D.; Feng, J.; Liu, X.; Feng, X. J. Org. Chem. 2015, 80, 9691. (c) Zhang, D.; Zhang, D.-M.; Xu, G.-Y.; Sun, J.-T. Chin. Chem. Lett. 2015, 26, 301. (d) Lu, Y.-L.; Sun, J.; Jiang, Y.-H.; Yan, C.-G. RSC Adv. 2016, 6, 50471.

(10) (a) Zhang, Y.; Wei, B.-W.; Lin, H.; Zhang, L.; Liu, J.-X.; Luo, H.-Q.; Fan, X.-L. Green Chem. 2015, 17, 3266. (b) Nagaraju, S.; Satyanarayana, N.; Paplal, B.; Vasu, A. K.; Kanvah, S.; Sridhar, B.; Sripadi, P.; Kashinath, D. RSC Adv. 2015, 5, 94474. (c) Lin, B.; Zhang, W.-H.; Wang, D.-D.; Gong, Y.; Wei, Q.-D.; Liu, X.-L.; Feng, T.-T.; Zhou, Y.; Yuan, W.-C. Tetrahedron 2017, 73, 5176. (d) Nagaraju, S.; Sathish, K.; Satyanarayana, N.; Paplal, B.; Kashinath, D. J. Heterocycl. Chem. 2020, 57, 469. (e) Nagaraju, S.; Satyanarayana, N.; Paplal, B.; Vasu, A. K.; Kanvah, S.; Sridhar, B.; Sripadi, P.; Kashinath, D. RSC Adv. 2015, 5, 81768. (f) Zhang, C.; Han, Y.; Ye, S. Chem. Commun. 2019, 55, 7966.

(11) (a) Chen, S.; Wang, G.-L.; Xu, S.-W.; Tian, M.-Y.; Zhang, M.; Liu, X.-L.; Yuan, W.-C. Org. Biomol. Chem. 2019, 17, 6551. (b) Liu, X.L.; Gong, Y.; Chen, S.; Zuo, X.; Yao, Z.; Zhou, Y. Org. Chem. Front. 2019, 6, 1603.

(12) Kartikey, K. D. D.; Reddy, M. S.; Chowhan, L. R. Tetrahedron Lett. 2020, 61, 152664.

(13) Nagaraju, S.; Sathish, K.; Paplal, B.; Satyanarayana, N.; Kashinath, D. New J. Chem. 2019, 43, 14045. 